\title{
Current Status of Intra-Vascular Imaging during Coronary Interventions
}

\author{
Sridhar Kasturi \\ Sunshine Heart Institute, Secunderabad, India \\ Email: sridharkasturi@yahoo.com,manikandhar@gmail.com
}

How to cite this paper: Kasturi, S. (2021) Current Status of Intra-Vascular Imaging during Coronary Interventions. World Journal of Cardiovascular Diseases, 11, 389-419. https://doi.org/10.4236/wjcd.2021.118038

Received: May 26, 2021

Accepted: August 27, 2021

Published: August 30, 2021

Copyright () 2021 by author(s) and Scientific Research Publishing Inc. This work is licensed under the Creative Commons Attribution International License (CC BY 4.0).

http://creativecommons.org/licenses/by/4.0/

\section{(c) (i) Open Access}

\begin{abstract}
Most important interventional application of IVUS is plaque assessment, vessel sizing and stent implantation guidance. Image guided PCI is associated with decreased incidence of stent thrombosis and MACE rate as evident in recently published meta-analysis of IVUS guided PCI studies. Many imaging studies have shown very clearly under-expansion, edge-dissection, tissue-prolapse, mal apposition, and geographical miss are associated with adverse events following DES implantation, and IVI guides in optimization of stent implantation by identifying and rectifying these predictors of DES outcomes.
\end{abstract}

\section{Keywords}

Left-Main Coronary Artery, Imaging, Intra-Vascular Ultra-Sound, Optical Coherence Tomography

\section{Introduction}

Conventional angiography is poor in assessing vessel wall, mainly characterization of plaque, plaque burden, circumferential and longitudinal distribution of the plaque, and features associated with suboptimal stent deployment. ICI provides cross sectional high resolution images of vessel wall high lighting morphology \& extent of plaque distribution, and helps in optimization of PCI by illustrating predictors of DES outcomes, namely under-expansion, mal apposition, and medial edge dissection after DES implantation [1].

OCT imaging has an axial spatial resolution of 10 to $20 \mu \mathrm{m}$, penetration depth of 1 to $2.5 \mathrm{~mm}$, and lateral resolution of $20 \mu \mathrm{m}$. Whereas, range of IVUS axial spatial resolution is about 100 to $200 \mu \mathrm{m}$, maximum penetration depth $-10 \mathrm{~mm}$, and lateral resolution $-200 \mu \mathrm{m}$. OCT is able to reveal more detail than IVUS due to its higher resolution but with limited depth assessment. A major drawback of 
OCT is the necessity of a contrast or saline or dextran for blood clearance, as near infrared light is fully attenuated by blood, and another limitation is the inability to obtain proper images of ostial lesions due to inadequate clearance of blood. OCT requires more usage of contrast medium for vessel flushing which might lead to increased risk of worsening renal function especially in patients with Diabetes, low GFR, and advanced age [2].

Most important interventional application of IVUS is vessel sizing and stent implantation guidance. Numerous studies have demonstrated IVUS guidance compared to CAG guidance results in large luminal dimensions thereby reducing the incidence of major adverse cardiovascular events. In the analysis of OPUS-CLASS study, IVUS imaging showed a large minimal luminal diameter and area than OCT by $9 \%$ and $10 \%$, respectively. In comparing between OCT and IVUS measurements in the phantom model, OCT was equal to the actual luminal area of the model while IVUS overestimated, angio underestimated the luminal area, and was less reproducible [3].

Plaque modification of severely calcified lesions with image guidance and usage of appropriate plaque modification devices improve stent expansion thereby reducing stent thrombosis and MACE rate. Image guided PCI is associated with decreased incidence of stent thrombosis and MACE rate as evident in recently published ULTIMATE [4], IVUS-XPL [5], ADAPT-DES [6] and meta-analysis of IVUS guided PCI studies. Many imaging studies have shown very clearly under-expansion, edge-dissection, tissue-prolapse, mal apposition, and geographical miss are associated with adverse events following DES implantation. In the latest guidelines of the European society of cardiology and European association for cardiothoracic surgery, OCT as well as IVUS is recommended for procedural optimization as class II a. OCT guidance is currently recommended by the EBC to support bifurcation lesion PCI and might expand to distal LMS lesion.

IVI should be considered for the evaluation of borderline and ambiguous lesions especially in cases of uncertain severity, very short lesions, pre aneurismal, or post aneurismal lesions, ostium of left main stenosis or RCA, branching lesions, sites with focal spasm or angiographically hazy lesions. OCT has limitation in identification of internal elastic lamina in severely diseased vessels which is likely to lead to smaller device selection compared to IVUS (Table 1).

IVI during PCI helps in Plaque assessment, vessel sizing, and stent implantation guidance. Preprocedural IVI helps in assessing plaque burden, type of plaque, and vulnerability to rupture, functional significance of the lesion, reference segments, stent size, landing zones, and helps in optimizing DES implantation by identifying and rectifying predictors of DES outcomes-stent under expansion/stent mal apposition, and medial edge dissection, tissue prolapse, intramural and extra mural hematomas (Figure 1) [7].

\section{Plaque Distribution and Assessment of Landing Zones}

IVUS assessment of plaque distribution with area of minimal luminal narrowing 
Table 1. Usefulness of intravascular imaging during coronary interventions.

\begin{tabular}{|c|c|}
\hline $\begin{array}{l}\text { Assessment of plaque } \\
\text { morphology }\end{array}$ & $\begin{array}{l}\text { Lipid plaque/fibrous plaque/fibro-calcific plaque/calcific plaque/luminal masses-red thrombus VS white } \\
\text { thrombus }\end{array}$ \\
\hline $\begin{array}{l}\text { Plaque modification } \\
\text { devices }\end{array}$ & NC balloons/high pressure OPN NC balloon/Scoring balloons/ROTA/OA/IVL/ELCA \\
\hline $\begin{array}{l}\text { For assessment of effectiveness } \\
\text { of plaque modification }\end{array}$ & Evidence of fractures/dissections on OCT/HD-IVUS \\
\hline Device selection & Device length \& Diameter \\
\hline For optimization of DES & Medial edge dissection/IMH/EMH/ expansion/apposition/tissue prolapse/LSD/geographical miss \\
\hline CTO lesions & Location of GW-intimal VS sub-intimal/IVUS guided antegrade puncture \\
\hline \multirow{2}{*}{ LM lesions } & Assessment of severity of LM by MLA, and decision about PCI strategy_single VS two-stent technique \\
\hline & Assessment of optimization of LM bifurcation result-expansion areas of LM, LM confluence, LAD \& LCX \\
\hline \multirow{2}{*}{ Bifurcation lesions } & Assessment of MLA, plaque burden \& extent of plaque length of MV/MB/SB \\
\hline & CT angle/BP-CT length (spiky carina, eye-brow sign) \\
\hline
\end{tabular}

\section{Intravascular imaging to understand disease severity and morphology}

Severity and distribution of calcification

Calcium Depth $>0.5 \mathrm{~mm}$, Arc $>180^{\circ}$, Length $>5 \mathrm{~mm}$ (need for ROTA/Cutting Balloon)

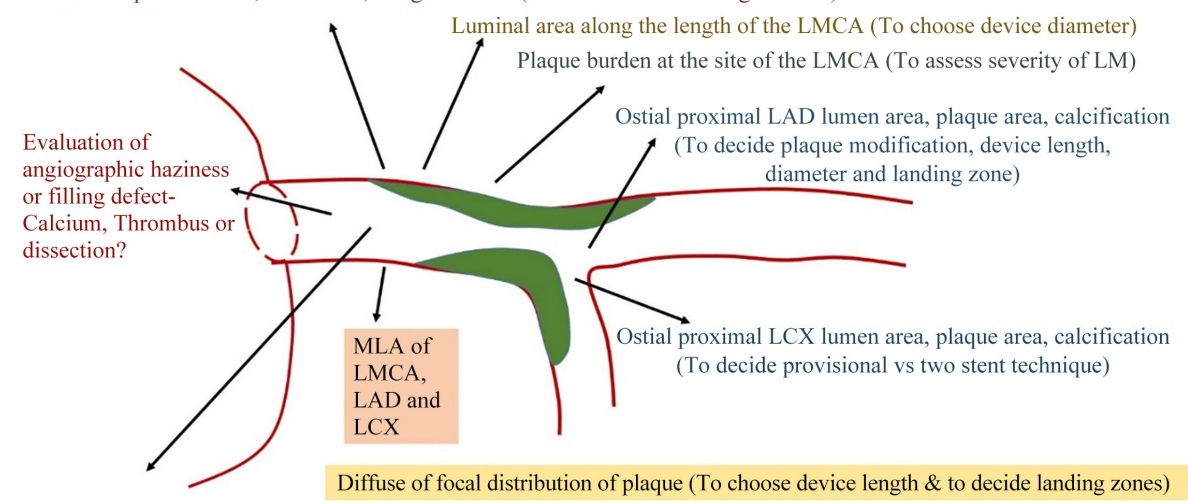

Figure 1. Intravascular imaging to understand disease severity and morphology.

at landing zones and reference segments is very important to decide for selection of proper stent diameter and length to cover entire diseased segment, and select a landing zone with a minimal plaque burden or disease free segment $5 \mathrm{~mm}$ either side of the stent edge in order to prevent future restenosis. Try to avoid landing zones with lipid rich plaques with TCFA, fibrotic plaque burden $>50 \%$, and calcific arc $>70 \%$. For selection of the stent size, choose size according to the distal reference landing zone diameter and upsize the proximal portion of the stent according to the proximal landing zone reference diameter by post dilating with appropriately sized balloon [8].

\section{IVUS and OCT Assessment of Normal Segments and Various Plaques}

Normal vessel: Three layered appearance of normal vessel, with the muscular 
media being revealed as a low single layer compromised between internal and external elastic lamina. Adventitia-The $3^{\text {rd }}$ and outer layer consists of the adventitia and peri-adventitial tissues. External elastic membrane is the outer layer of the vessel wall in IVUS measurement because the border of adventitia and peri-adventitial tissue is not distinct (Figure 2(a)). Intimal leading edge can be easily identified because the intima was thickened enough to be resolved as a separate layer and has sufficiently different acoustic impedance from lumen in normal segments. In normal vessels and at the sites of thin plaques, with thicknesses not exceeding $1.2 \mathrm{~mm}$, the coronary artery wall appears as a three-layer structure in OCT images. Intima: signal-rich layer nearest the lumen; media: signal-poor layer in middle of vessel wall (thickness ranges from 125 to 350 micro meter-mean 200 micro meter), adventitia: signal-rich outer layer of vessel walls (Figure 2(b)) [9] [10]

A plaque was defined as a region with a loss of the three-layered structure (ie, intima, media, and adventitia) of the vessel wall (Figure 3).

Soft plaque-Mainly lipid deposit, echo lucent in nature, not as bright as adventitia (hypoechoic). Plaques may be eccentric or concentric based on distribution of atheroma (Figure 4). CLIMA study involving more than 1000 patients identified certain features suggestive of high risk plaques on OCT-intimal cap thickness of $<75 \mu \mathrm{m}$ findings, stenosis severity long lipid rich plaque, and macrophages as predictors of the future adverse hard clinical events, death and target lesion related myocardial infections [11].

EROSION trial showed that majority of patients (92.5\%) with ACS caused by plaque erosion who were managed with aspirin and Ticagrelor without stenting remained free of major adverse cardiac events for less than 1 year and conservative medical management may be an alternative option in these patients [12].

(a)

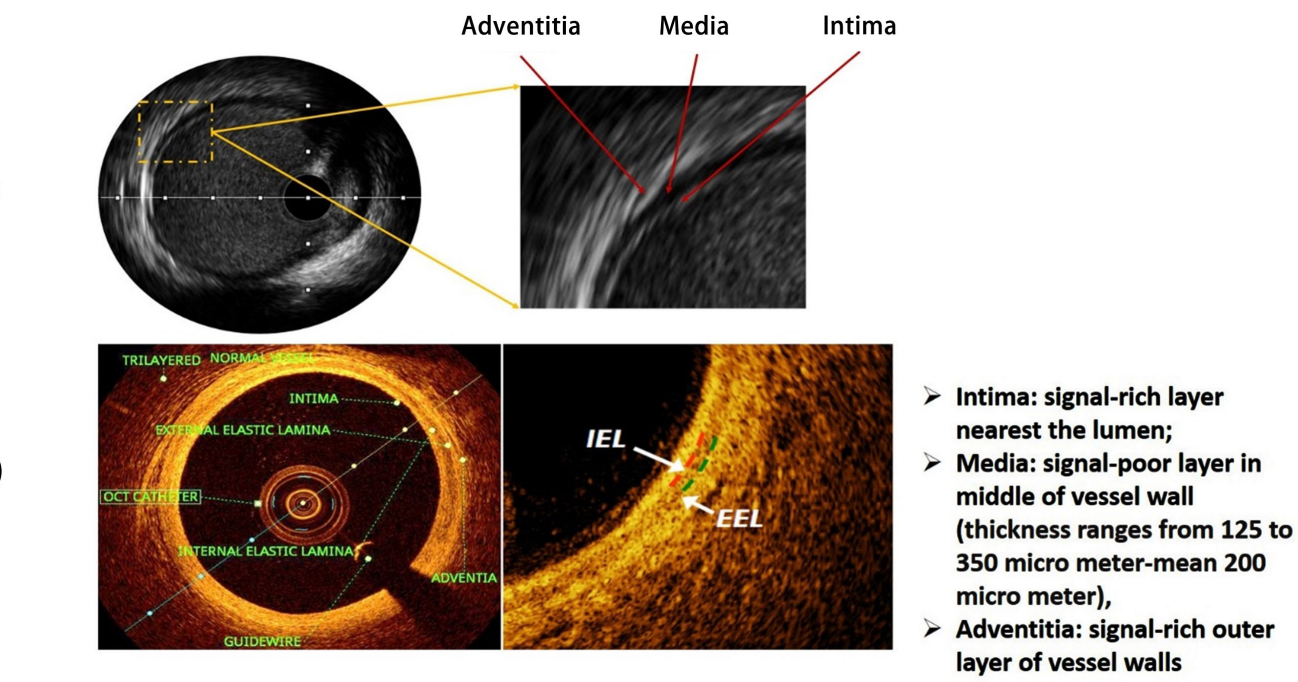

$>$ Intima: signal-rich layer nearest the lumen;

(b)

The coronary artery wall appears as a three-layer structure in OCT images.

Figure 2. Normal vessel by IVUS and OCT. 


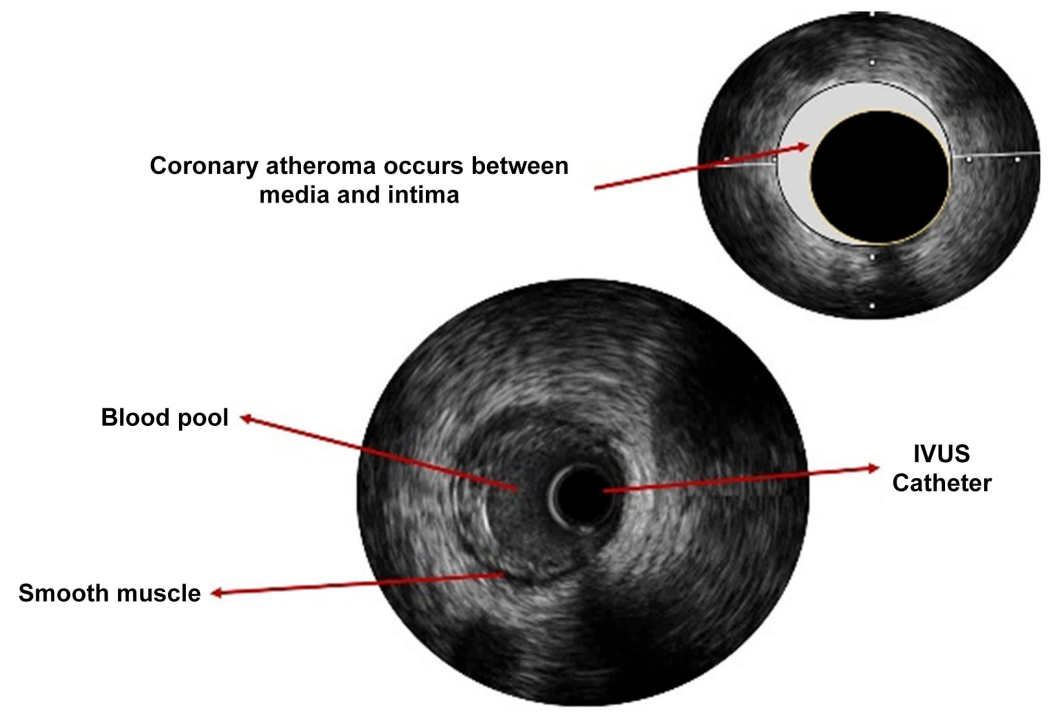

Blood, fat and smooth muscle reflect ultrasound poorly

Figure 3. Coronary atheroma by IVUS.

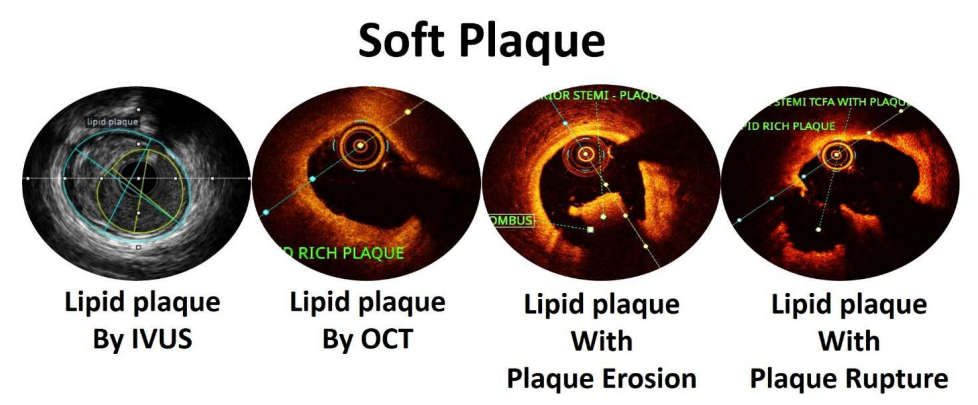

Figure 4. Lipid plaque identification by IVUS and OCT, Lipid rich plaque with plaque rupture and erosion.

Fibrotic plaque-Collagen rich fibrous tissue produces dense and bright echoes without acoustic shadowing which is as bright or bright as the adventitia (hyperechoic). Fibrotic tissue partially reflects ultrasound but does not cause signal dropout (Figure 5).

Calcific plaque-As seen as a signal-poor or heterogeneous region with sharply delineated leading, trailing, and/or lateral borders. High echo brighter (Bright white) than adventitia obstructs the penetration of ultrasound (acoustic shadowing-Signal dropout behind) and reflects ultrasound rays, hence only the leading edge is detected and thickness cannot be determined [13]. IVUS is useful to assess calcium 1) Location-Superficial Calcium vs Deep Calcium, 2) The Arc of Calcium (in degree), 3) The Length of the Calcified Deposit (Figure 6). Calcific plaque can be classified as 1) Single arc of calcium (90 degrees), 2) Two arcs calcium (180 degrees), 3) Three arcs (270 degrees), and 4) Circumferential (360 degrees) based on circumferential extent of calcium plaque (Figure 7, Table 2).

Calcium is described qualitatively according to its location: lesion versus reference and superficial (leading edge of acoustic shadowing within the shallowest $50 \%$ of the plaque and media thickness) versus deep (leading edge of acoustic 
shadowing within the deepest $50 \%$ of the plaque and media thickness). The sensitivity was $86.7 \%$, the specificity was $93.3 \%$, and the predictive accuracy was 92.3\% for detecting calcium as a signal-poor or heterogeneous region with sharply delineated leading, trailing, and/or lateral borders [13].

\section{Eccentric fibrous}

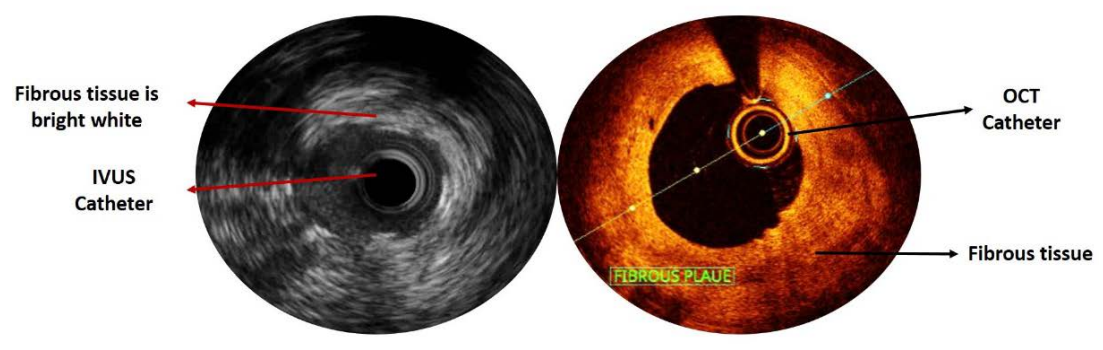

Does not cause signal dropout, as it is fibrotic material, not dense calcification

Figure 5. Fibrous plaque identification by IVUS and OCT.
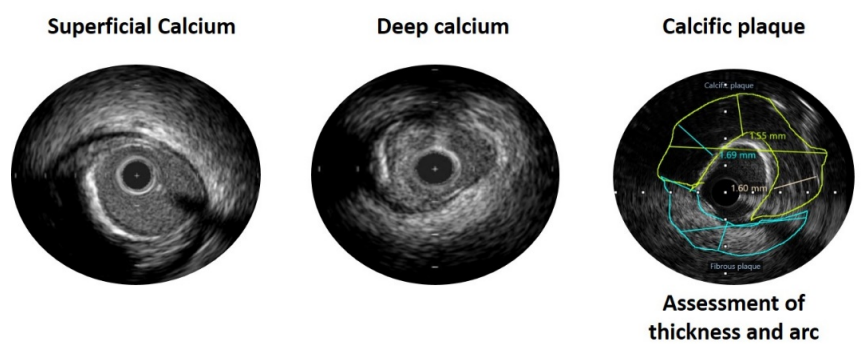

Calcium Nodule
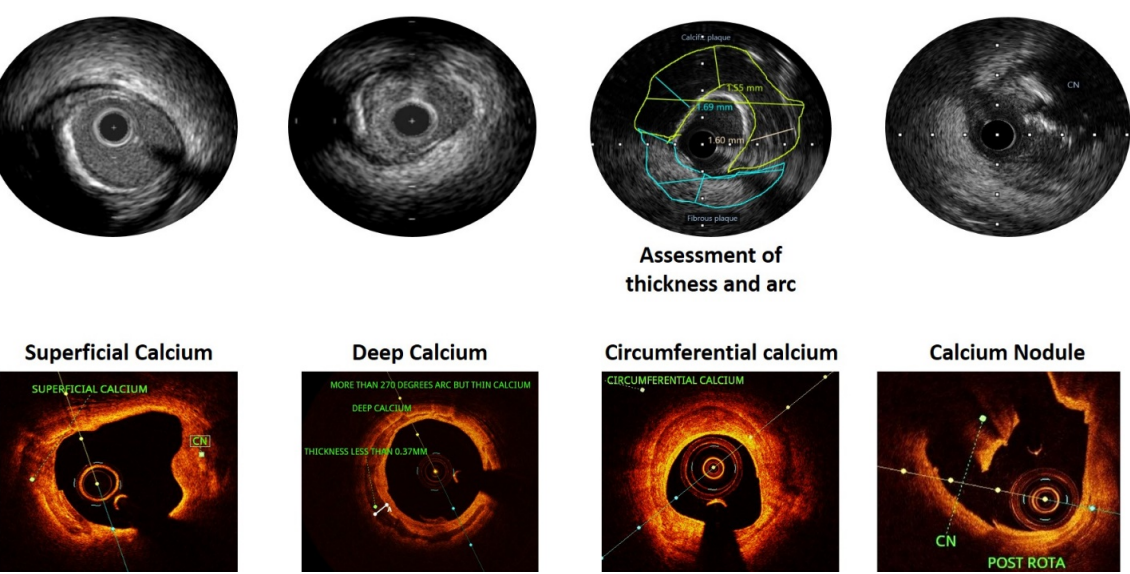

Calcium Nodule

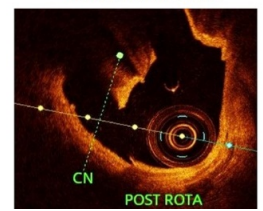

Figure 6. Types of calcific plaque by IVUS and OCT.

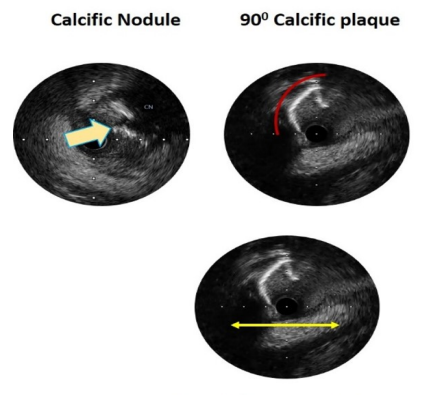

Vessel diameter $-4.5 \mathrm{~mm}$

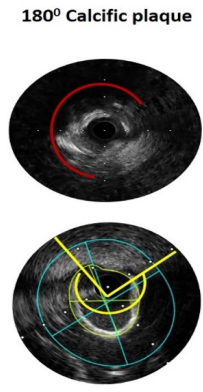

Calcium angle $-270^{\circ}$
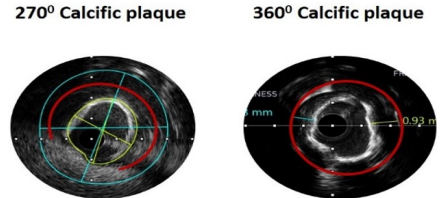

Reverberations

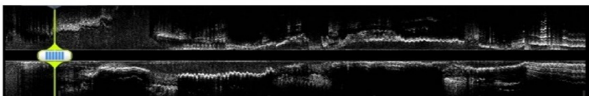

IVUS assessment of Calcium according to the arc (measured in degrees, using a protractor cantered on the lumen) and length (using motorized transducer pullback). Semi quantitative grading classifies calcium as absent or subtending 1, 2, 3, or 4 quadrants

Figure 7. Calcific plaque classification by IVUS. 
Table 2. According to calcified arc in IVUS and OCT images it can be divided into V degree.

\begin{tabular}{cc}
\hline 0 Degree & No \\
I Degree & within $90^{\circ}$ \\
II Degree & $91^{\circ}-180^{\circ}$ \\
III Degree & $181^{\circ}-270^{\circ}$ \\
IV Degree & $271^{\circ}-360^{\circ}$ \\
\hline
\end{tabular}

If plaque assessment reveals severely calcified lesions, assess its severity, calcium expansion index, various types of calcification, and any need for plaque modification devices. If post ROTA/IVL/OA, OCT shows superficial calcium thickness $<0.5 \mathrm{~mm}$ and calcium fractures, no need to up size the plaque modification device or additional modification device, and it indicates adequately modified calcium plaque which results in adequate stent expansion. Inadequately prepared calcium plaque requires up size of device or additional modification device, otherwise it might lead to procedure delay, difficulty in delivering stents, stent dislodgement, balloon rupture, vessel perforation, and under-expansion which are associated with acute and long-term MACE rate.

Mixed plaque-Associates with soft and dense plaque, produces bright echoes with or without acoustic shadowing. Concentric plaques are distributed circumferentially in the vessel; concentric plaques tend to occur in areas of negative remodeling (Figure 8) [14].

IVI should be used to assess plaque size, plaque length, plaque type, and helps in choosing stent size to cover entire diseased segment, and any need for plaque modification strategy prior to stenting to avoid under expansion, mal apposition, and edge related problems like medial edge dissection and geographical miss.

\section{Usefulness of IVI in Identifying Remodelling}

Remodelling refers to changes in vascular dimensions during the progression of atherosclerosis. It can be either positive (when the vascular area increases as plaque develops) or negative (when the vascular area decreases as plaque develops). 1 Intravascular ultrasound (IVUS) assesses arterial remodelling by comparing the lesion external elastic membrane (EEM) with the reference segments to generate a remodelling index (RI) [15].

Positive remodelling-Diameter of vessel at lesion site/ diameter of vessel at proximal reference $>1.05$ (Figure 9).

Negative remodelling-Diameter of vessel at lesion site/ diameter of vessel at proximal reference $<0.95$ (Figure 9).

\section{Remodelling Index}

The remodeling index is calculated as the EEM cross sectional area at the MLA divided by the average of the proximal and distal reference EEM CSA (Figure 9). 


\section{Basic IVUS measurements}

Choosing reference segments-choose most normal looking area (site with largest lumen with minimal plaque burden, an average having 35\% to 50\% plaque burden on IVUS) within $5 \mathrm{~mm}$ from the lesion site (maximum stenosis), and no intervening major side branches (Figure 10).

\section{Stent sizing (Table 3):}

Selection of stent diameter should be based on mean EEM or luminal diameter of distal landing zone, and length of the stent to cover entire diseased segment extending from proximal landing zone to distal landing zone, and choose landing zones with a normal segment or reasonably healthy zone with a plaque burden of $<50 \%$ (Figure 11 ).

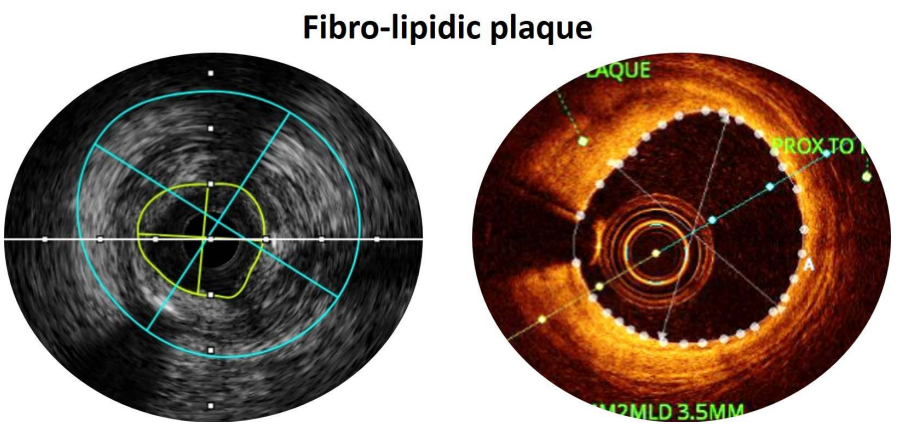

Figure 8. Mixed plaque is a combination of tissues of varying echogenicity.

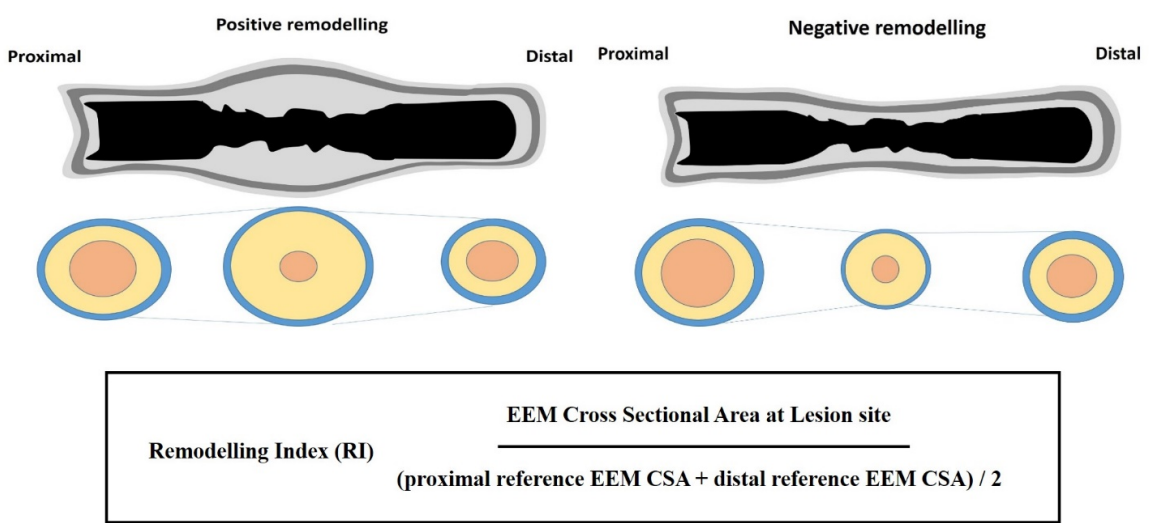

Figure 9. Positive and negative remodelling along with RI.

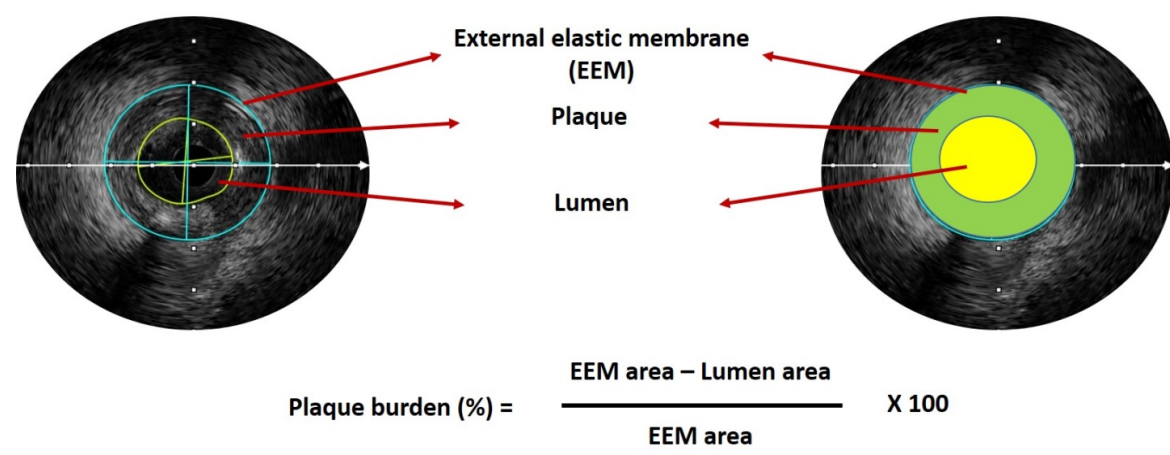

Figure 10. Plaque burden by IVUS. 


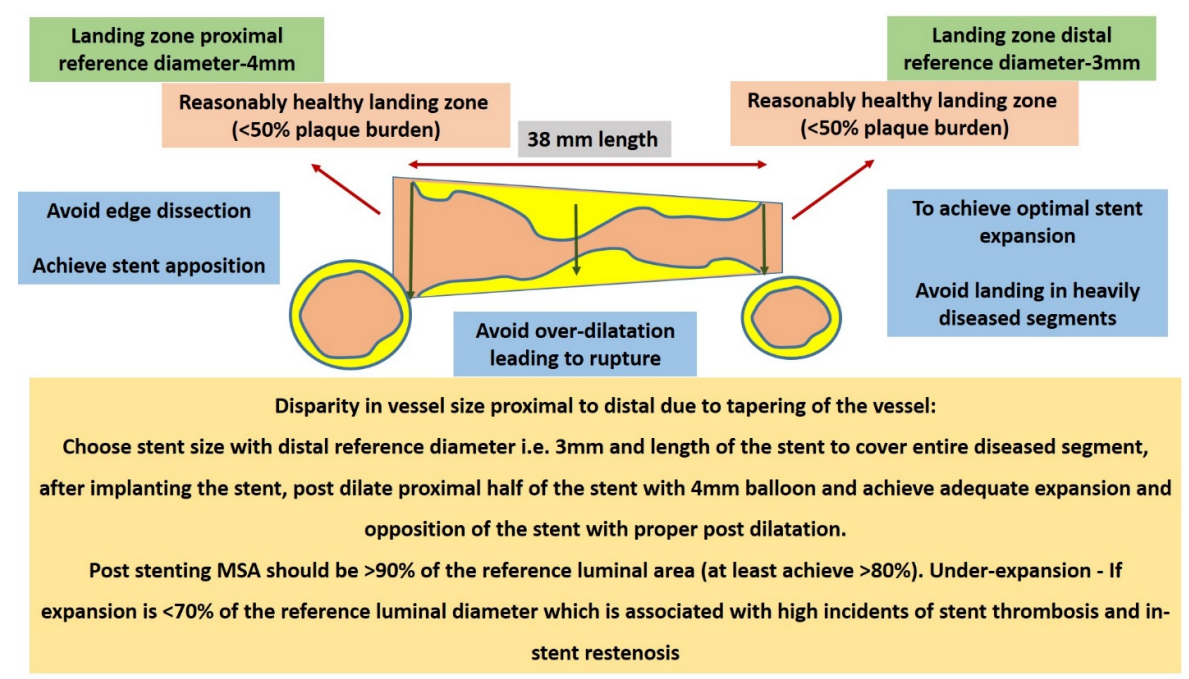

Figure 11. Stent sizing guideline.

Table 3. Criteria for optimal expanded stent.

\begin{tabular}{|c|c|}
\hline Study & Criteria for optimal expanded stent \\
\hline Katrisis & Full stent apposition, MSA $\geq 90 \%$ mean MLA symmetrical expansion ( $\min \mathrm{SD} / \mathrm{max} \mathrm{SD}>0.7$ ) (correlation with FFR 0.94) \\
\hline Fearon & MSA $7 \mathrm{~mm}^{2}$ (correlation with FFR 0.94 ) \\
\hline Hanekamp & $\begin{array}{l}\text { Full stent apposition, MSA } \geq 90 \% \text { mean MLA or MSA } \geq 100 \% \text { min MLA symmetrical expansion ( } \min \mathrm{SD} / \mathrm{max} \mathrm{SD}>0.7 \text { ) } \\
\text { (correlation with FFR } 0.94 \text { ) }\end{array}$ \\
\hline Gorge & symmetrical expansion, MSD $\geq 3 \mathrm{~mm}$, full apposition \\
\hline Choi & Complete stent apposition, MSA $\geq 80 \%$ distal MLA symmetrical expansion ( $\min \mathrm{SD} / \mathrm{max} \mathrm{SD}>0.8$ ) \\
\hline Colombo & Full stent apposition, MSA $\geq \min$ MLA MLA $>60 \%$ MLA in edge regions \\
\hline Ahmed & Complete stent apposition, MSA $\geq 80 \%$ mean MLA or MSA $7.5 \mathrm{~mm}^{2}$ \\
\hline \multirow{4}{*}{ MUSIC } & $\begin{array}{l}\text { - Complete stent apposition, MSA } \geq 90 \% \text { mean MLA or } \geq 100 \text { min MLA } \\
\text { - In case of }>9 \mathrm{~mm}^{2}:\end{array}$ \\
\hline & MSA $\geq 80 \%$ mean MLA or $\geq \min$ MLA \\
\hline & MSA in proximal part of the stent $\geq 90 \%$ prox MLA \\
\hline & Symmetrical expansion $(\operatorname{minSD} / \operatorname{maxSD}>0.7)$ \\
\hline Blasini & Full stent apposition, MSA $>8 \mathrm{~mm}^{2}$, or MSA $\geq 90 \%$ mean MLA, complete coverage of dissection \\
\hline RESIST & MSA $\geq 80 \%$ mean MLA \\
\hline OPTICUS & MUSIC criteria, residual stenosis $<10 \%$ (angiographically) \\
\hline CRUISE & Residual stenosis $<10 \%$ (angiographically) \\
\hline AVID & Full stent apposition, residual stenosis $<10 \%$, no signs of dissection \\
\hline TULIP & Complete stent apposition, MSD $\geq 80 \%$ mean MLD, MSA $\geq 90 \%$ distal MLA \\
\hline
\end{tabular}

Post stenting IVUS guidance should be used, to make sure the stent is fully expanded with good apposition, and the stent covered entire plaque length. Otherwise the recurrence of ISR or late stent thrombosis will be very high.

Stent expansion-Stent expansion describes the minimum stent cross-sectional area either as an absolute measure (absolute expansion), or compared with the predefined reference area, which can be the proximal, distal, largest, or average reference area (relative expansion). Stent expansion is calculated as MSA divided 
by the average reference lumen area multiplied by 100 . The reference lumen area is determined from the most normal-looking slice with the reference segment, which is defined as a slice having the largest lumen area, because the external elastic membrane is not always visible by OCT compared to IVUS. Aim for achieving good expansion of stent preferably more than $90 \%$ of reference mean luminal area. If stent expansion area is $<70 \%$ compared to distal or proximal reference area, it is considered as under expansion, which is associated with ST and ISR. Under expansion should be corrected with high pressure inflation of appropriate sized NC balloon. If it persists despite NC balloon dilatation, it needs OPN NC balloon or IVL or both to address deep calcium behind stent struts to get adequate expansion of stent. In some cases of severe ISR due to under-expansion with underlying severe calcification plaque modification with Rota might be considered (Figure 12).

Mal apposition-Stent mal-apposition (or ISA) is defined as separation of the stent struts from the vessel lumen wall in a region not overlying a side branch. Stent mal-apposition and under-expansion can co-exist or occur independently. Stent mal apposition is considered as significant when Stent adjacent vessel lumen distance is $>0.3 \mathrm{~mm}$ with longitudinal extension $>3 \mathrm{~mm}$, and it is associated with stent thrombosis and difficulty in crossing with guide wires due to ab-luminal placement of wires. Major mal apposition should be corrected with appropriately sized NC balloon, and minor mal appositions can be treated conservatively.

Edge dissection-Significant medial edge dissections are with linear rim of tissue with a width $>0.2 \mathrm{~mm}$ and a clear separation from the vessel wall or underlying plaque that was adjacent $(<5 \mathrm{~mm})$ to a stent edge. MED with longitudinal extension $>2 \mathrm{~mm}$, lateral extension $>60^{\circ}$ and involvement of deeper layers (medial or adventitia) are considered large dissections which require additional stenting to cover the dissection (Figure 13).

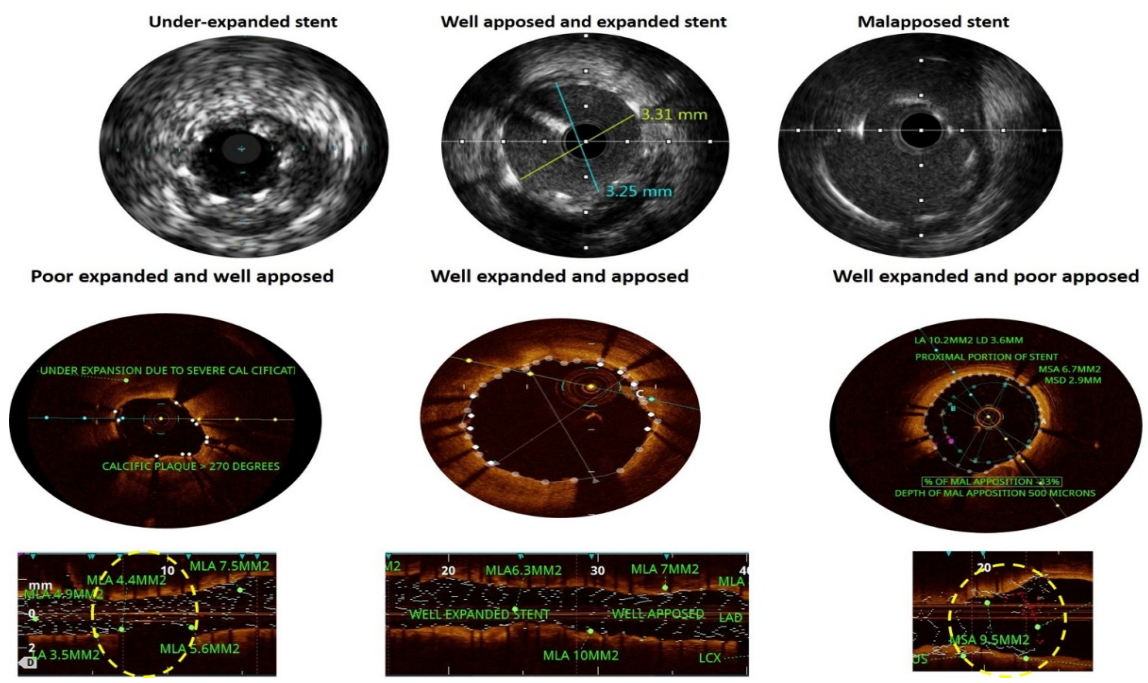

Figure 12. Identification of under expansion and malapposition varying from well expansion and apposition of stent struts. 


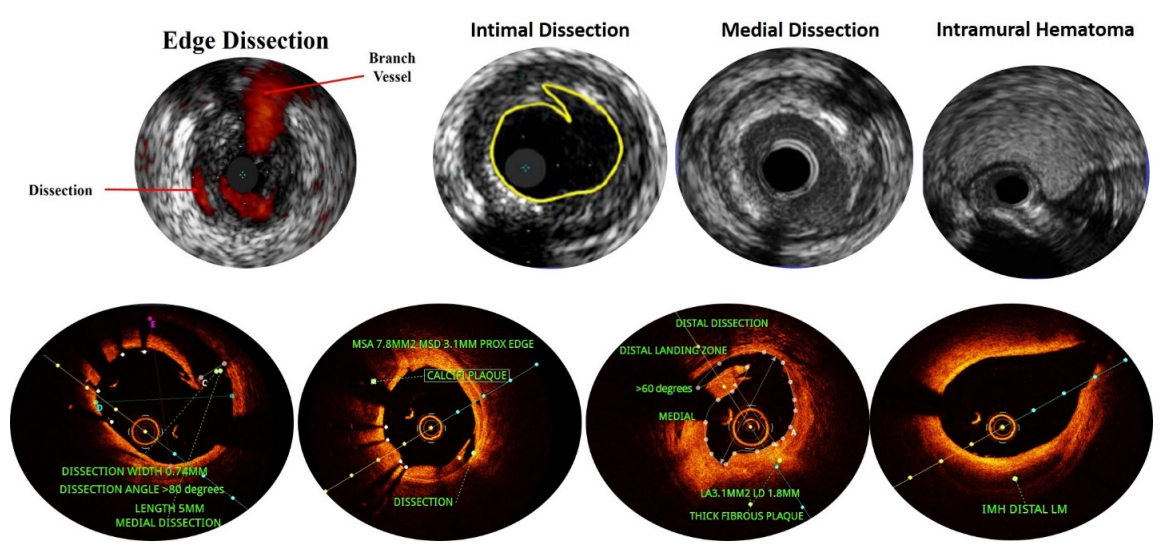

Figure 13. Stent edge dissection and intramural hematoma by IVUS and OCT.

Tissue prolapse-Tissue prolapsing (TP) between stent struts extending inside a circular arc connecting adjacent struts or intraluminal mass $>0.5 \mathrm{~mm}$ in thickness with no direct continuity with the surface of the vessel wall or highly backscattered luminal protrusion in continuity with the vessel wall and resulting in signal free shadowing. Small tissue protrusion, small tissue prolapse does not require any treatment, and major tissue prolapse are resistant to treatment. TP should be distinguished from thrombus, and large irregular tissue prolapse associate with increased MACE rate (Figure 14).

Reference luminal narrowing-Lumen area $<4.5 \mathrm{~mm}^{2}$ in the presence of significant plaque adjacent to stent endings.

In stent minimal luminal cross sectional area (MLA) - In stent MLA $<4.5$ $\mathrm{mm}^{2}$ and $70 \%$ of the average reference lumen area is considered significant which requires further expansion with upsized balloon.

Gatto et al. observed stent MLA $<4.5 \mathrm{~mm}^{2}$ and narrowing of references were the more common features of suboptimal stent deployment identified with OCT. Angio-co registration of OCT system which reflects the location of the OCT camera on the coronary angiogram is useful to choose stent length, landing zones and minimize geographic miss [16].

As per the recommendations of an expert consensus document of the European Association of Percutaneous Cardiovascular Interventions (2018), identify following predictors of DES outcome and rectify them to provide better acute and long term outcomes following DES implantation (Figure 15) [17].

The most relevant targets to be achieved following stent implantation in non-LM lesions are shown. These include optimal stent expansion (absolute as well as relative to reference lumen diameter); avoidance of landing zone in plaque burden $>50 \%$ or lipid rich tissue; avoidance of large mal apposition regions, irregular tissue protrusion, and dissections.

IVI is more useful to evaluate patients with stent failure; it provides various causes of stent failure to decide appropriate treatment. Stent restenosis may be due to neo-intimal growth, neo-atherosclerosis, under-expansion, and disease progression in the stent borders. The OCT is superior to IVUS and should be the imaging modality of choice to assess stent failure (ST and ISR) because it is 
much more precise to determine underlying pathology.

Stent Thrombus The presence of a thrombus that originates in the stent or in the segment $5 \mathrm{~mm}$ proximal or distal to the stent. OCT identifies stent thrombus as intraluminal lesion with irregular edges, oscillation at periphery, low density, differentiates white thrombus from red thrombus and while assessing consideration of underlying clinical situation is more important (Figure 16).

OCT assessment of stent failure mechanisms is essential to determine best therapeutic strategy-Stent edge restenosis should be treated with additional stent, Neo atherosclerosis or neointimal hyperplasia can be managed with additional stent or $\mathrm{DEB}$, under-expansion or mal apposition require post-dilatation with NC balloons, and plaque modification is required in patients with neo-atherosclerosis with severe calcification or calcific plaque is responsible for under expansion.

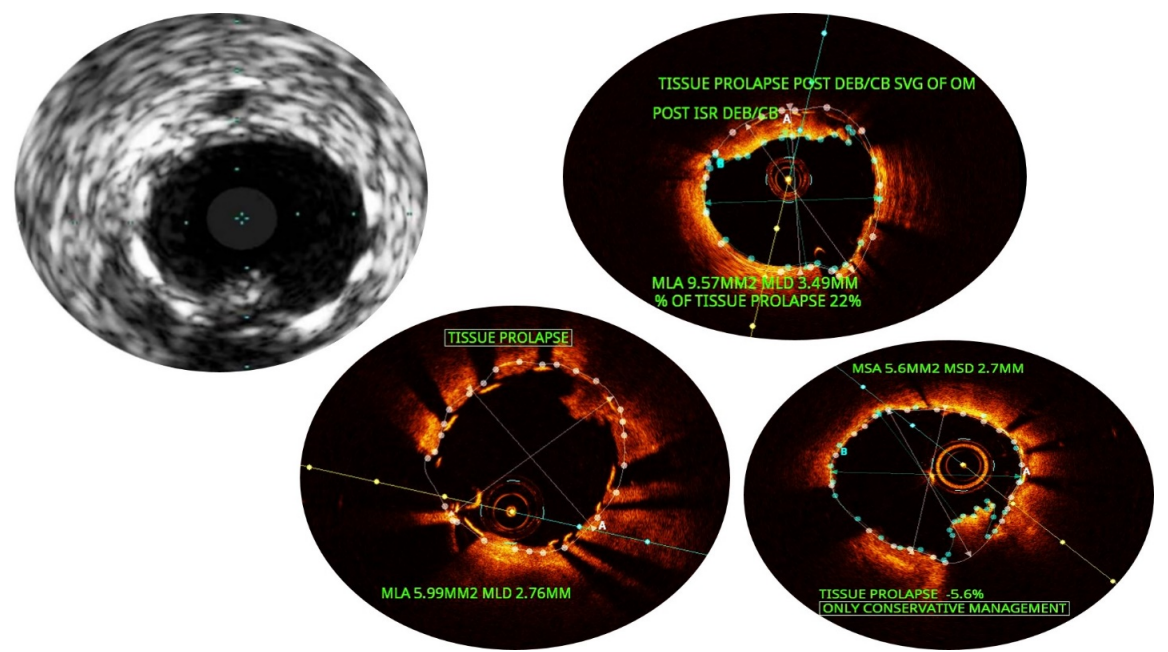

Figure 14. Tissue prolapse identification by IVUS and OCT.

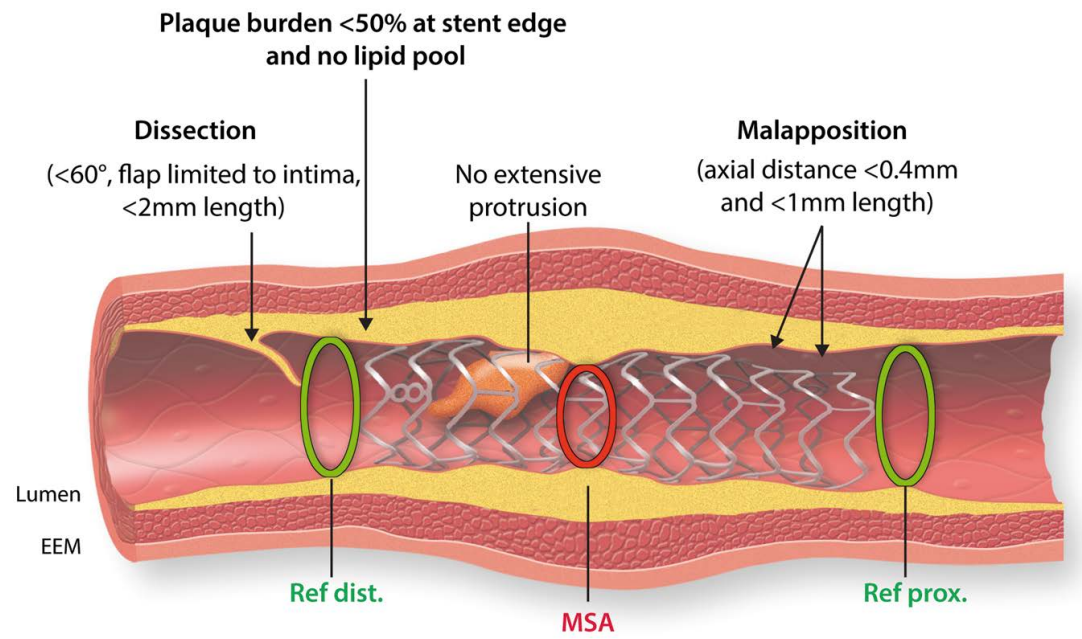

MSA $>5.5 \mathrm{~mm}^{2}$ (IVUS) and $>4.5 \mathrm{~mm}^{2}$ OCT

MSA/average reference lumen $>80 \%$

Figure 15. Predictors of drug eluting stent outcomes. 


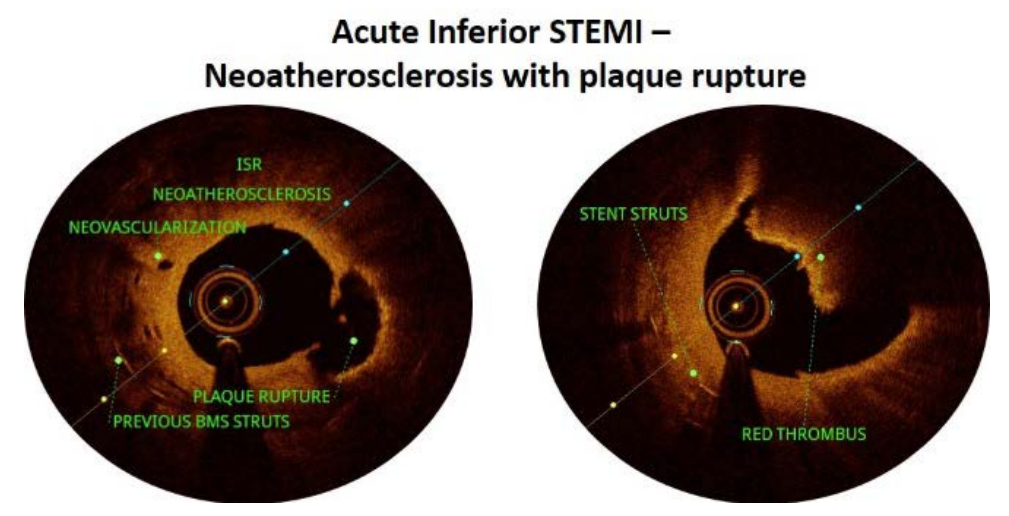

Figure 16. Neo-atherosclerosis with plaque rupture.

Stent Restenosis-In-Stent Restenosis is defined as the gradual re-narrowing of a stented coronary artery lesion due to arterial damage with subsequent Neo-intimal tissue proliferation or due to development of Neo-atherosclerosis with lipid or fibro-lipid or calcific intima (Figure 17).

Differentiates True and false lumen-IVUS and OCT can help confirm wire position in the true or false lumen, the true lumen is often compressed by the false lumen (Figure 18).

OCT has a limited a penetration depth, it is very difficult to identify plaque burden especially in lipid rich plaque. In contrast, calcified plaque can be visualized well with OCT, whereas IVUS is not capable of penetrating calcified plaques. Therefore, IVUS should be preferred for assessing the plaque burden and vessel size in patients presenting with lipid rich plaques, whereas OCT should be preferred for calcified plaques. OCT has much higher resolution compared with IVUS and should therefore be considered for lumen assessment and stent related morphology, such as thrombosis, restenosis, edge dissection, expansion and mal apposition.

\section{IVI Assessment of Bifurcation during the Procedure}

Intravascular ultrasound imaging during the procedure allows for precise: 1) direct control of wire re-crossing through the jailed SB, 2) assessment and optimisation of stent apposition (stent strut adherence to the vessel wall), 3) measurement and optimisation of stent expansion (ratio between minimum stent area and lumen area in adjacent reference segment of the vessel), 4) assessment of full lesion coverage by the stent (especially at the SB ostial location when using some double-stenting techniques such as T-stenting), and 5) diagnosis and treatment of stent edge problems (geographic miss, secondary lesions, large plaque burden, dissection, etc.). IVI pullbacks in both SB and MV are recommended in evaluation of two-stent techniques if intracoronary imaging is used.

OCT assessment of guide wire re-crossing point, link connection and jailing struts on the SB ostium using 3D OCT image provides some more insights during bifurcation PCI. If suboptimal guide wire re-crossing and SB ostial dilatation or stent mal apposition or deformation is found, and an additional optimization 


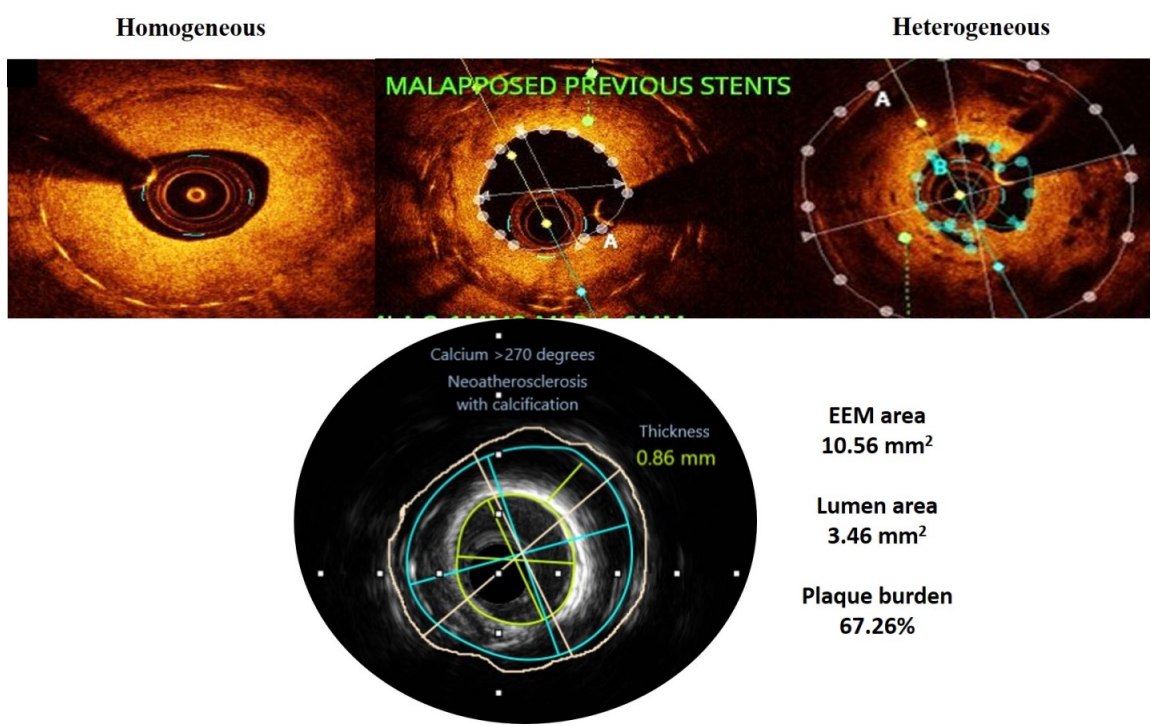

Figure 17. In-stent restenosis by OCT and IVUS.

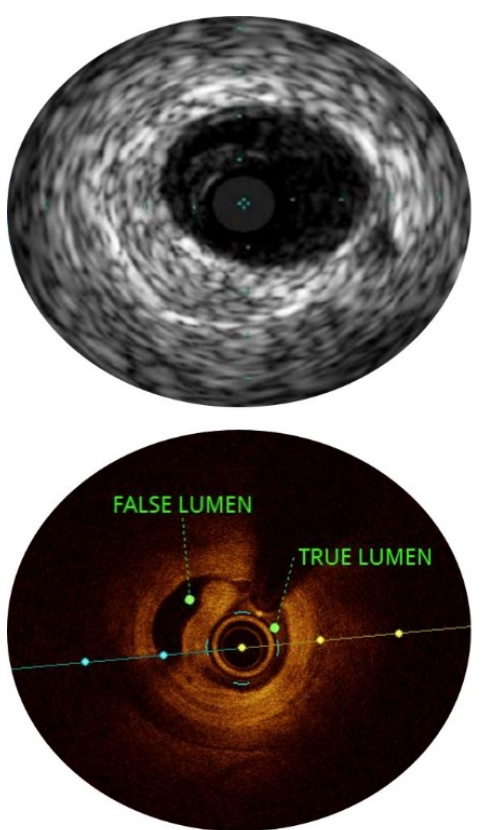

Pre stent assessment
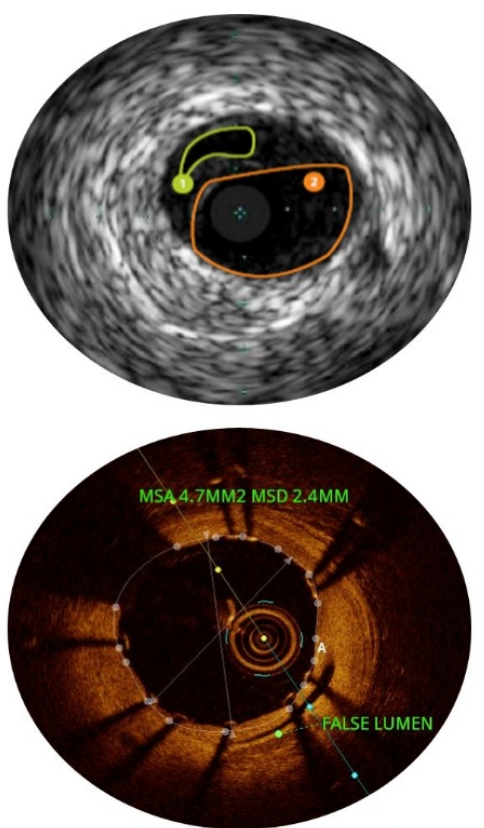

Post stenting compressed false lumen

Figure 18. True and false lumen identification by IVUS and OCT.

procedure is recommended. Incomplete blood flushing in large vessels or shadow of guide wire in OCT is prone to inaccurate assessment of mal apposition or guide wire re-crossing position.

\section{Importance of IVUS Guided Optimization of LM-PCI}

LM has more elastic component that may lead to stent under expansion and recoiling, more often associated with calcific plaques with higher probability of vessel tapering. LM bifurcation disease is mostly diffuse and angiography may be inaccurate in assessing the disease severity of ostia of both branches. Whereas, 
IVI provides more accurate information by providing cross sectional images about plaque morphology, disease extent, severity, and size of the LMCA, LCX, and LAD vessels. Presence or absence of significant disease in the ostium of the LCX is an important factor in selecting a stent strategy (single Vs two stents). If unable to pass imaging catheter due to severe calcification or fibrosis of LM lesion needs pre dilatation with small sized balloon or plaque modification, and usage of guide extension catheter for placement of IVUS/OCT catheter. Angio assessment of Post LM PCI with two-stent technique is unreliable in detecting under-expansion and mal apposition, and IVI is the best method to show under-expansion and mal apposition which are associated with significant MACE rate and correction of these by IVI improves acute and long term outcomes [18]. Angiographic ISR is more frequent in lesions with under-expansion than without $(24.1 \%$ vs $5.4 \%)$. In the 2 stent group, the lesions with complete expansion of all sites showed a restenosis of only $6 \%$, similar to that in the single stent group (6.3\%). Post-stenting under-expansion was an independent predictor of 2-year MACE, especially repeat revascularization. 34\% of patients of LM PCI showed under-expansion of at least one of the segments of LM bifurcations (LM, POC, LAD, LCX), and most often affects LCX ostium which was observed in $37 \%$ of patients. Under-expansion of at least one of the pre-specified segments was more frequent with the 2-stent group (54\%) vs the one stent group (27\%). Kang et al. evaluated IVUS predictors of in-stent restenosis (ISR) after LM bifurcation stenting. Post-stenting IVUS MSA cut-offs that best predicted ISR on a segmental basis were $5.0 \mathrm{~mm}^{2}$ (Ostium of left circumflex [LCX] ISR), $6.3 \mathrm{~mm}^{2}$ (ostium of left anterior descending [LAD] ISR), $7.2 \mathrm{~mm}^{2}$ (ISR within polygon of confluence [POC, confluence zone of LAD and LCX]), and $8.2 \mathrm{~mm}^{2}$ (ISR within the LM above the POC) (Figure 19). A smaller IVUS-MSA within any one of these segments was responsible for a higher rate of angiographic ISR and clinical major adverse cardiovascular events (MACE). Edge restenosis was predicted by residual plaque burden of $>51.6 \%$ to $54.5 \%$ in the DES stented segment [19].

OCT has some theoretical advantages over IVUS, including: 1) an improved detail and resolution of superficial intra vascular structure prior to intervention, 2) a complete analysis of stent apposition and expansion and final MLA assessment, and 3) the ability to discover tissue prolapse, thrombus or edge dissection after stent implantation. OCT guidance for LM PCI was feasible, safe and was successful in $86 \%$ of patients in LEMON study [20].

\section{Usefulness of IVI to Anticipate Side Branch Compromise}

Side branch (SB) ostial stenosis can be aggravated after crossover stent deployment in the MV, the main mechanism for the SB compromise was carina shift, pre-procedural $\mathrm{PB}$ and luminal expansion after stenting of the distal MV were associated with the carina shift7 which is enhanced by stretching of the distal MV and a shift in the flow divider outward towards the SB ostium [21]. Presence of negative remodelling in the distal MV, particularly calcified plaque in the MV opposite to the SB orifice was a predictor of SB residual stenosis after FKB. 


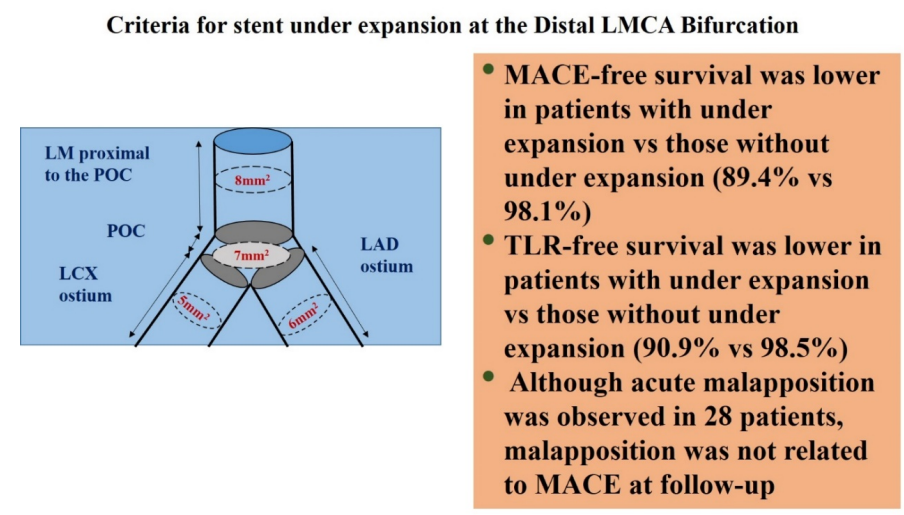

Figure 19. Criteria for stent under expansion at the distal LM bifurcation.

Therefore, selection of an appropriately sized stent (according to the precise lumen diameter at the MV reference and at the negative remodelling carina site) via pre-procedural IVUS/OCT is an important technical step for avoiding unnecessary SB occlusion after MV stenting. Subsequent proximal optimisation technique (POT) can correct any proximal stent mal apposition that is due to the luminal discrepancy between the proximal and distal MV. Pre-procedural minimal lumen area of $<3.7 \mathrm{~mm}^{2}$ and $\mathrm{PB}>56 \%$ at the left circumflex (LCX) ostium predicted a post-stenting FFR $<0.80$ after LM bifurcation stenting with the crossover technique [22].

OCT assessment of Carina tip (CT) angle, bifurcation point (BP) angle, BP-CT length is helpful to predict SB closure before provisional stenting. SB closure more likely if $\mathrm{CT}$ angle $<51^{\circ}$, BP-CT length $<1.75 \mathrm{~mm}$, and if more plaque burden of MV/SB at the bifurcation site [23]. The parallel type of bifurcation lesions in which proximal course of SB is concealed behind the carina in the 3D perpendicular image of SB more likely to be associated with carina shift compared to perpendicular type in which proximal SB visualized over the carina.

In provisional stenting prior to FKB it would be preferable to cross through the distal strut which ensures better scaffolding of SB ostium with stent struts, guide wire through proximal strut prior to $\mathrm{FKB}$ is associated with mal apposition and improper scaffolding of SB ostium. In two stent technique OCT guides about the position of guide wire proximal vs distal strut to minimize mal apposition and better expansion with adequate scaffolding of SB ostium with stent struts.

\section{OCT Assessment of Predictors of SB Closure}

The assessment of stent configuration over SB orifice and guide wire re-crossing portion with 3D OCT imaging before KBI provides important information to achieve optimal bifurcation strategy. Proper POT enlarges the distal site of jailed struts, which increase the likelihood of optimal distal wiring. Stent configuration is classified into two types-1) link free carina type, (No link connection on the carina) - Distal guide wire re-crossing is required to provide better stent opposi- 
tion to the lateral wall after KBI. 2) Link connecting carina type, (Link connection is location between the carina and proximal stent strut)-no difference in stent apposition regardless of guide wire re-crossing position, a KBI with distal guide wire re-crossing in the link connecting type has potential risk of stent deformation (Figure 20).

Angio-guided PCI with stenting of distal LM with mild lesions of proximal LM are likely to be underestimated, and if stent is not extended up to the ostium of LM, increased chances of getting proximal edge restenosis, and IVI is best method to assess severity of LM and helps in deciding proper landing zone to reduce proximal edge restenosis. Takagi et al. showed the efficiency of the combination of POT and full coverage of ostial LM on the reduction of ISR in ostial LM group compared to propensity score-adjusted group that was not treated with this strategy [24].

Stent struts at ostial LCX after provisional stenting impacted the narrowing of the Ostial area at follow up OCT study and main pathological predictors for LM stent failure are mal apposition and struts crossing an ostial LCX supported the importance of the opening the stent struts jailing the SB. The 3D OCT imaging facilitates the achievement of complete removal of jailed struts and fully apposed struts in the bifurcation segment, which may lead to improvement of KBI compared to 2D OCT imaging or angio guidance.

In patients with bifurcation PCI with provisional stenting (PS) an additional two stent strategy may be necessary in $3 \%-47 \%$ of cases after provisional stenting. In PS, SB dissection and bail out two stent deployed occurred in $10.5 \%$ and $5.6 \%$ after KBI, respectively, even when dedicated IVUS guided KBI was performed. Imaging guidance is useful to avoid unnecessary bail out two stenting in PS which will reduces MACE rate.

\section{Assessment of the Ostium and Lesion Length of SB}

Properly assess plaque burden of SB ostium and lesion length by pull back study of IVUS/OCT of SB and MB to MV. This will be useful in selecting proper landing zones, stent length and diameter if two stent technique required. If SB is not significantly diseased avoid SB stenting.
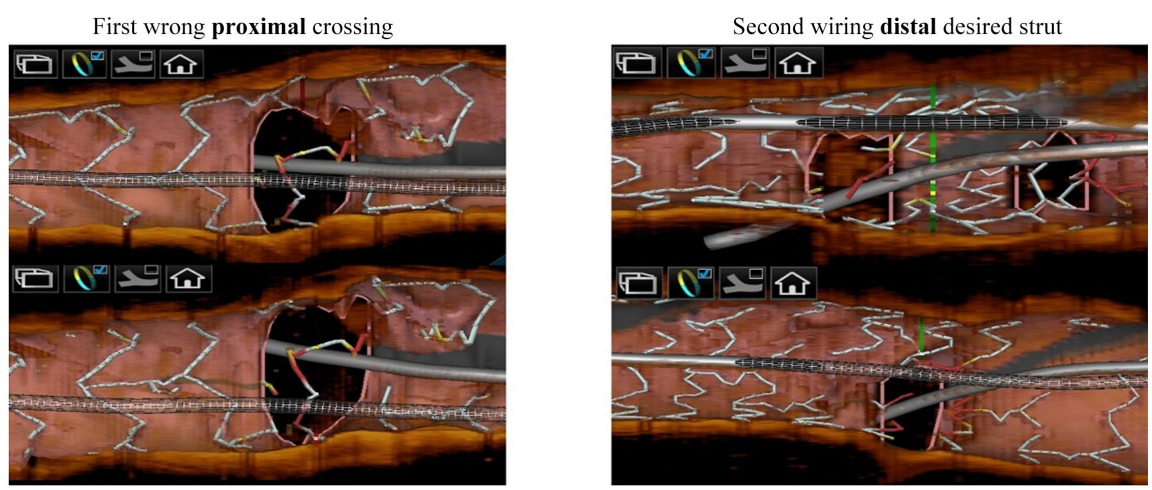

Figure 20. Wiring of side branch through proximal struts vs distal struts. 


\section{IVUS Assessment of Severity of LMCA Disease}

Atherosclerotic plaque of the LMCA is qualitatively different from else-where in coronary tree, with minimal necrotic core content and less thin fibro-atheroma than the proximal segment of the other coronary arteries particularly the LAD, which is more prone for ruptures [25]. Conventionally an angiographic cut off of $>50 \%$ diameter stenosis (equivalent to $>75 \%$ area stenosis) has been used to indicate hemodynamic significance, which is on the bases of early work in an animal model by Gould that demonstrated a reduction in hyperemic flow across lesions beyond this disease degree of stenosis [26].

Oveido et al. demonstrated that LM disease extending into the proximal LAD, LCX or both may be seen in $90 \%, 66.4 \%$ and $62 \%$ of patients, respectively, whereas ostial daughter vessel lesions without LMCA involvement was present in only $9.3 \%$ of LAD and $17.1 \%$ of LCX vessels, and the carina was always spared [27].

Fractional flow reserve (FFR) is the gold standard for identifying myocardial ischaemia (FFR $\leq 0.80$ ) and to determine the functional significance of LM bifurcation [28]. To predict myocardial ischaemia in LM disease, the cut-off value for the IVUS-derived minimum lumen cross-sectional area (IVUS-MLA) has been recognised as $4.5-6.0 \mathrm{~mm}^{2}$ [29]. The IVUS MLA in LM seems to be population-dependent. The average IVUS MLA in the patients included in two different studies was strikingly different $\left(7.6 \mathrm{~mm}^{2}\right.$ in the US study and $4.8 \mathrm{~mm}^{2}$ in the Korean study) [30]. In a prospective multicentre LITRO study, investigators evaluated the safety of an IVUS-MLA threshold of $6 \mathrm{~mm}^{2}$ to guide decision making on revascularization in 354 patients with LM disease.

In 179 patients with an LM segment IVUS-MLA of $\geq 6 \mathrm{~mm}^{2}$ revascularization was deferred, whereas the remaining 152 patients with an IVUS-MLA $<6 \mathrm{~mm}^{2}$ were revascularized. Importantly, the two-year event-free survival from cardiac death and MI was $97.7 \%$ in those patients who had no LM revascularization, compared with $94.5 \%$ in the revascularized group [31]. Recent ESC guidelines proposed that LM patients (Figure 21) with an IVUS MLA of $<4.5 \mathrm{~mm}^{2}$ should be recommended for revascularization, IVUS MLA between $>4.5 \mathrm{~mm}^{2}$ and $<6$ $\mathrm{mm}^{2}$ should be evaluated with FFR, if FFR positive need revascularization and negative needs to be treated conservative medical management, and if IVUS-MLA of $\geq 6 \mathrm{~mm}^{2}$ revascularization of LMCA should be deferred [32].

OCT is considered as non-applicable for coronary arteries ostia and might be limited in case of large vessel but it scores over IVUS in identification of thrombus, coronary dissection and in complete stent opposition due to its better spatial resolution. It is comparable to IVUS in assessing mid and distal LMs specific features such as diameter discrepancies, tapered anatomy, plaque eccentricity and high probability of calcification that are difficult to correctly analyze by angiography alone.

OCT image acquisition may not be possible in extremely tight and unstable lesions, requiring pre-dilatations to get adequate blood free field with contrast 


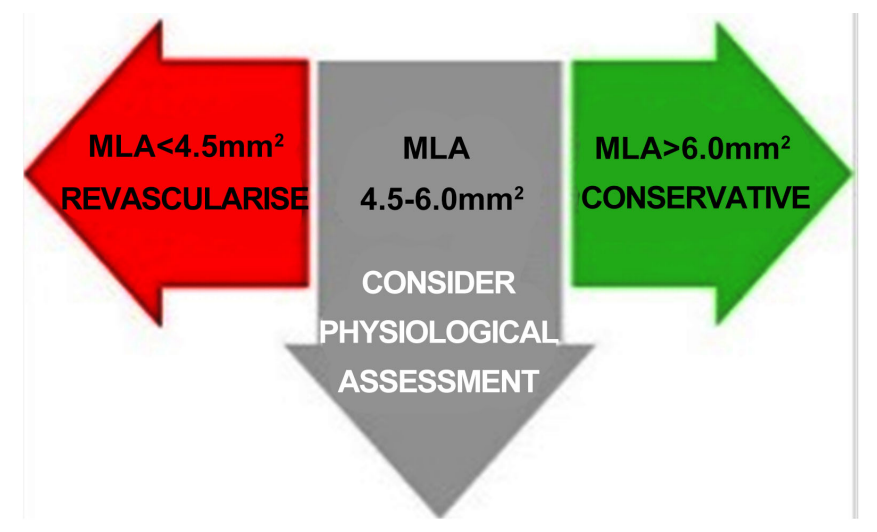

Figure 21. Consideration of LM area by IVUS.

injections which is essential in obtaining good images, and optimal OCT imaging occurs in vessels $<4.5 \mathrm{~mm}$ in diameter, while the range of LMCA diameter is 3.5 to $5.5 \mathrm{~mm}$.

Unfavorable results reported in some trails of PCI of LMCA lesions may be due to use of 1st generation stents and various technical issues; guide catheter induced dissections, under expansion, uncovered diseased segments, multiple mal apposed stent strut, dense jailing of Ostium of SB, longitudinal stent deformation and accidentally crushed stent may all contribute to worse outcomes. Pre dilatation of areas that will not be covered with stent should be avoided. Pre dilation of LCX prior to stenting the main vessel with subsequent rewiring of the pre-dilated SB may increase the risk of the guidewire entering dissections, may influence outcomes adversely.

\section{OCT Assessment for Crush Evaluation}

In crush techniques, post crushing OCT run shows very clearly about the length of crushed segment in the MV and adequacy of crushed segment of SB stent proximal portion in MV. If crush is inadequate due to under sized balloon, upsizing of balloon is required to do repeat crushing to ensure crushing is adequate prior to implanting MB-MV stent (Figure 22).

\section{OCT Assessment for Stent Deformation}

OCT runs after stent implantation can show stent deformation either shortening or longitudinal by showing multiple layers of overlapped stents at the site of deformation. Most often observed at the proximal edge of the stent due to guide catheter, old NC balloon, big sized balloons hitting the stent edge while performing pulling and pushing manipulations particularly when there is no proper alignment and with jailed wire in situ (Figure 23).

Protrusion of stent into the aorta during stenting of LM ostium may interfere with engagement of guide catheter, and may increase risk of crushing of the stent. Immediate expansion and opposition may be of importance to avoid collision with guide catheter and lower the risk of LSD. 


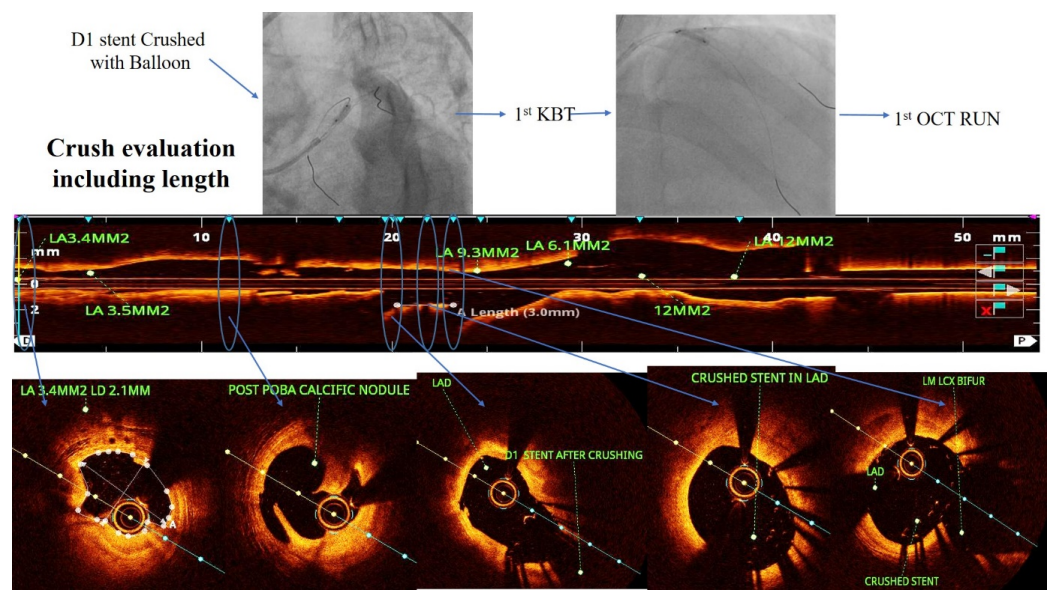

Figure 22. Crush evaluation by OCT.

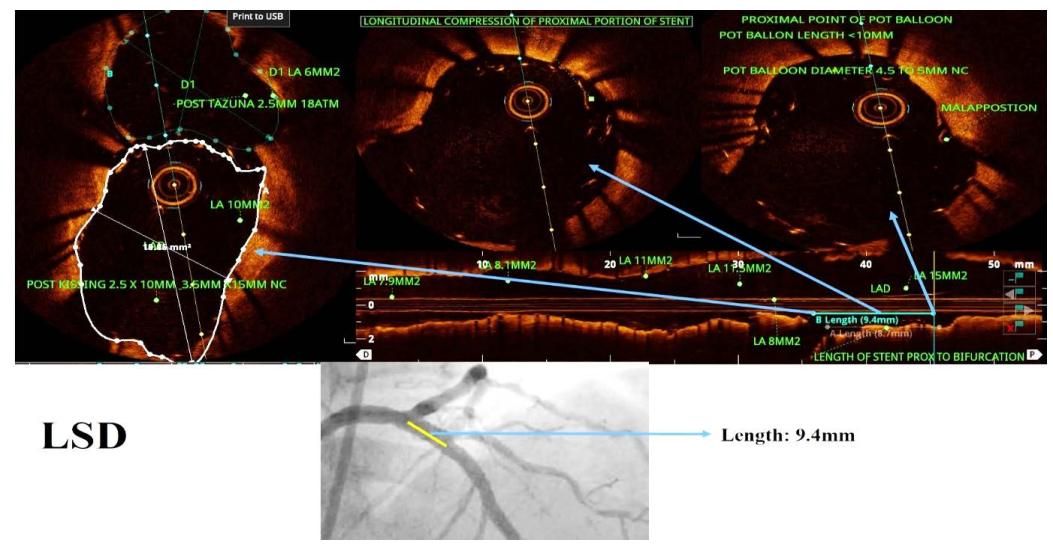

Figure 23. Longitudinal stent dislodgement by OCT.

\section{Usefulness of IVUS in CTO Interventions}

IVUS evaluation gives longitudinal information of CTO segment for proper entry point, and cross-sectional information of intimal plaque, sub-intimal space, true lumen and false lumen, and any vessel perforation, extravasation, sub-intimal dissection while tracking guidewire across long segment complex CTO lesions (Figure 24).

\section{Antegrade Approach of CTO}

IVUS in SB at the site of CTO is helpful for antegrade puncture of proximal blunt cap by confirming guidewire location, intimal vs sub-intima. IVUS guided re-entry from subintimal space during antegrade dissection and re-entry after parallel wire failure and when no retrograde option is possible. IVUS is also helpful after STAR with long subintimal segment, by advancing IVUS in false lumen and try to re-enter intima with stiffer wire (Figure 25) by showing an example of a procedure (Figure 26(a) \& Figure 26(b)).

\section{IVUS in Retrograde Approach of CTO}

IVUS is very useful to clarify retrograde wire location and the exact point of 
re-entry, in Reverse CART it is very useful for balloon sizing and to understand where is possible to try to make the connection between antegrade and retrograde wires, and permits to optimize stent sizing and expansion in complex coronary reconstruction (Figure 27).

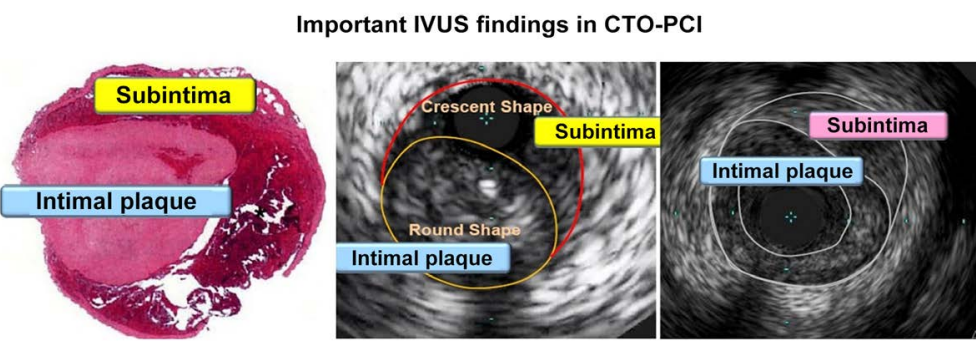

(a)

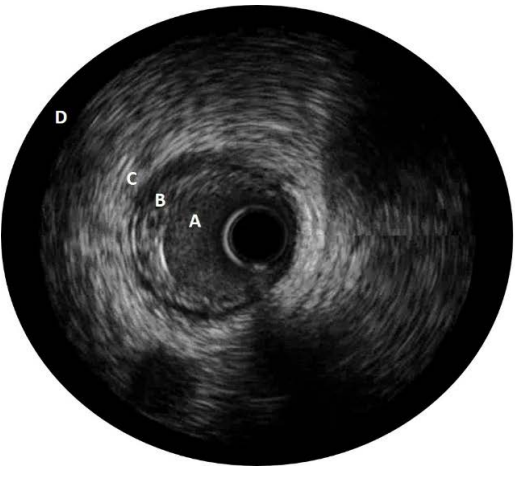

IVUS identifies location of a guidewire - anywhere within the three layers of the target vessel (Figure AIntima, B-Media, C-Adventitia) or outside of the vessel (i.e. in the pericardial space: Figure-D)

(b)

Figure 24. (a) Usefulness of IVUS in CTO segments; (b) Identification of guidewire by IVUS.

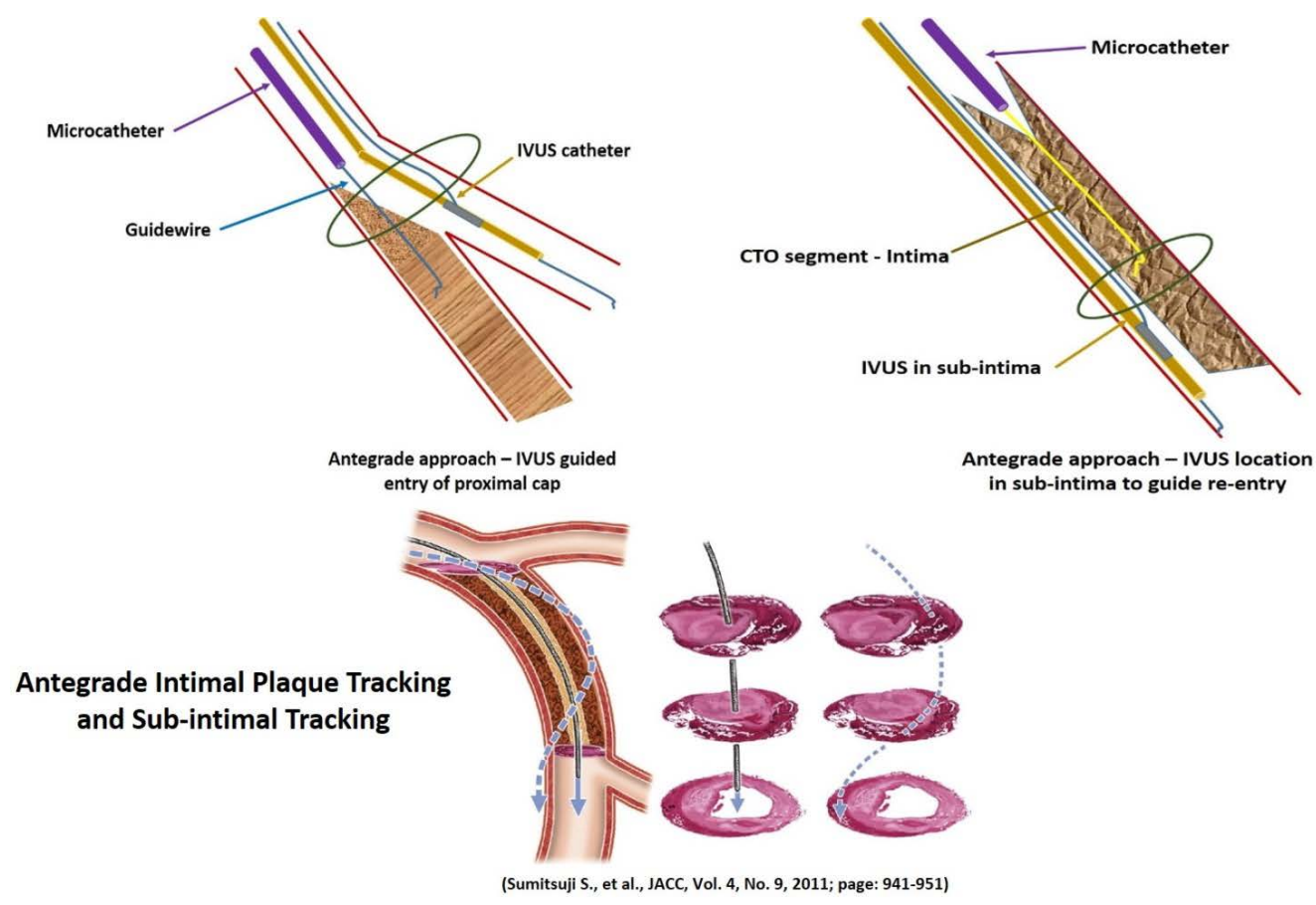

Figure 25. CTO antegrade approach. 

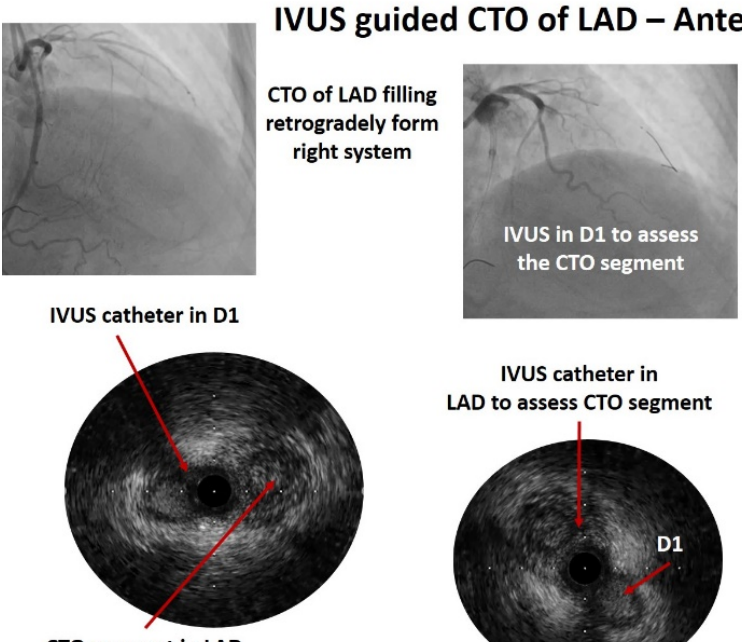

CTO segment in LAD

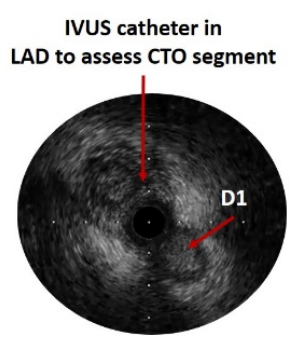

(a)

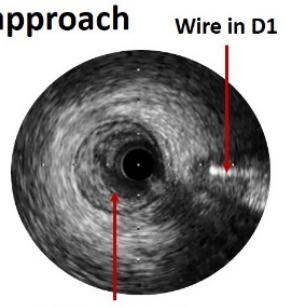

IVUS catheter in Distal LAD to assess CTO segment

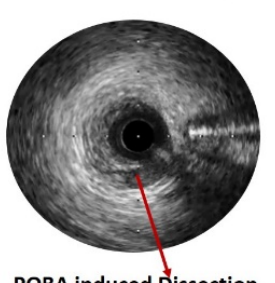

POBA induced Dissection

Micro catheter injection of LAD

Well expanded LM-LAD 3X30mm Orsiro stent
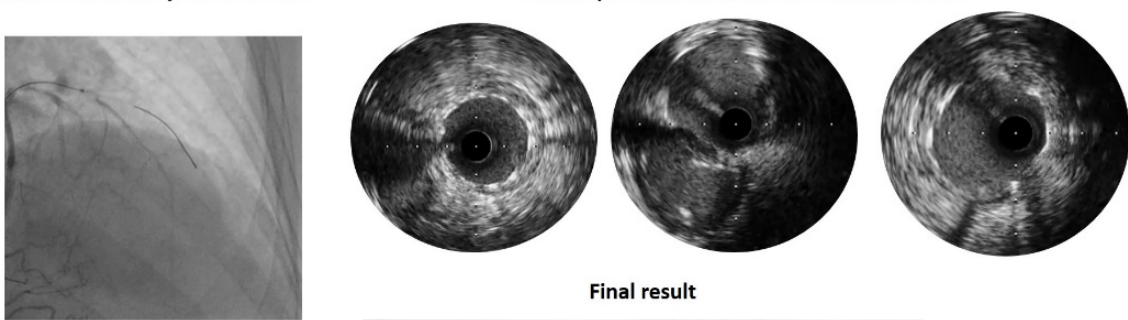

Final result

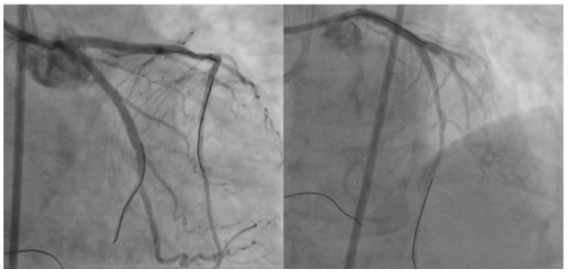

(b)

Figure 26. (a) IVUS guided antegrade CTO approach pre-stenting; (b) IVUS guided antegrade CTO approach post-stenting.

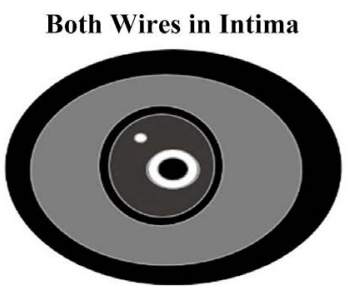

Both Wires in sub Intima

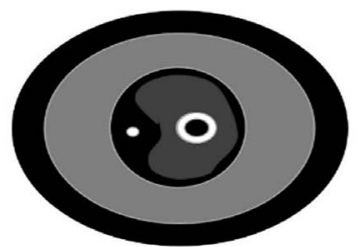

Antegrade Wire in Intima \& Retrograde Wire in Sub-Intima
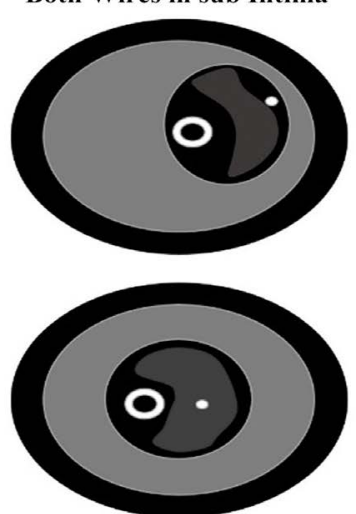

Antegrade Wire in Sub-Intima \& Retrograde Wire in Intima

Figure 27. IVUS in CTO by retrograde approach. 


\section{IVUS Identifies Location of Antegrade and Retrograde Wires during Retrograde PCI}

It is also helpful in identifying dissection planes in distal bifurcations, eliminates the need for antegrade injections prior to stenting thereby reduces risk of propagation of iatrogenic dissection distally or retrograde in the aortic wall (ostial RCA CTO) [33] and reduces the risk of CIN by reducing the volume of contrast particularly in Diabetics, elderly, and in patients with low GFR.

\section{Does IVI Change PCI Outcomes?}

Recent meta-analysis of 26,503 patients from three randomised and 14 observational studies (including two studies dedicated to LM stenosis and three studies dedicated to bifurcation stenosis). Intravascular ultrasound-guided PCI was significantly associated with more, longer and larger stents. Regarding clinical outcomes, IVUS-guided PCI was associated with a significantly lower risk of death, MI, ST and TLR [34] (Table 4).

\section{IVUS Guidance Saves Lives in LM PCI Compared to Angio Guided PCI}

Image guided PCI associated with decreased incidence of stent thrombosis and MACE rate which is evident in recently published Meta-analysis, ADAPT-DES [6], ULTIMATE [4], and IVUS-XPL [5] study. The ADAPT-DES [6] study showed improved clinical outcomes, both acute ( $<30$ days) and 1 year follow up. IVUS sub study at 1 year was associated with 33\% reduction in MI, 50\% reduction on ST, and $38 \%$ reduction in ischemic driven TLR. IVUS guided group tend to use longer stent length, bigger stent size, optimization or change in procedure in $70 \%$ of cases with angio guidance. In the MAIN-COMPARE registry [38], there was a tendency of lower risk of three-year mortality with IVUS guidance compared to angiography guidance (6.0\% vs. $13.6 \%)$. In particular, in patients receiving DES, the three-year incidence of mortality was lower with IVUS guidance as compared to angiography guidance (4.7\% vs. $16.0 \%)$. The incidence of definite and probable ST was significantly lower in the IVUS group $(0.6 \%$ vs. 2.2\%). Finally, IVUS-guided revascularization was identified as an independent predictor of major adverse events in the overall population (hazard ratio: 0.70 , 95\% CI: 0.52 to 0.99 ), and to a greater extent in the subgroup with distal lesions (hazard ratio: $0.54,95 \% \mathrm{CI}: 0.34$ to 0.90 ). CLI-OPCI study of 670 patients showed superiority of OCT guidance over angiography in terms of cardiac death and $\mathrm{MI}$ at 1 year (6.6\% v/s 13\%) [39]. More recently, OPINION trial showed OCT guided PCI was non-inferior to IVUS guided PCI in the terms of TVF $5.2 \%$ vs $4.9 \%$ at 12 months follow up, and it included bifurcation lesions in $38 \%$ of cases, revealed no statistical difference in TVF between IVUS and OCT guidance [40]. The ILLUMIEN III trial is a randomized study compared to an OCT or IVUS or angiography guided angioplasty. It proved the non -inferiority of OCT v/s IVUS regarding the MSA obtained [41] (Table 5). 
Table 4. Subgroup analysis was performed to assess the benefit of IVUS-defined optimal procedure over suboptimal procedure.

\begin{tabular}{llllll}
\hline Trials & $\begin{array}{l}\text { Sample } \\
\text { Size (n) }\end{array}$ & Centre & Key Inclusion Criteria & $\begin{array}{l}\text { Follow-up } \\
\text { (Months) }\end{array}$ & Primary Endpoint \\
\hline CTO-IVUS 2015 [35] & 201 & Multi-centre & CTO & 12 & Cardiac death: $0 \%$ versus $1 \%, \mathrm{p}=0.16$ \\
Tan et al. 2015 [36] & $61 / 62$ & Single centre & Unprotected LM & 24 & MACE: $13.1 \%$ versus $29.3 \%, \mathrm{p}=0.031$ \\
IVUS-XPL 2015 [5] & 700 & Multi-centre & Long lesions (stent length $>28 \mathrm{~mm}$ & 12 & MACE: $2.9 \%$ versus $5.8 \%, \mathrm{p}=0.007$ \\
Zhang et al. 2016 [37] & 42 & Single centre & Small vessel disease $(2.25-2.75 \mathrm{~mm}) 12$ & $\begin{array}{l}\text { Post-PCI MLD: } 2.77 \pm 0.46 \mathrm{~mm} \text { versus } \\
\text { ULTIMATE 2018 [4] }\end{array}$ \\
\hline & 72 & Multi-centre & All corners & 12 & TVF: $2.9 \%$ vs 5.4\%, $\mathrm{p}=0.019$ \\
\hline
\end{tabular}

Table 5. Comparison of IVUS and OCT.

\begin{tabular}{|c|c|c|}
\hline Pre procedure & IVUS & OCT \\
\hline Co-registration with angiogram & ++ & ++ \\
\hline Sizing of vessel & ++ & + \\
\hline Sizing of lumen & ++ & ++ \\
\hline Assessment of plaque distribution & ++ & + \\
\hline Plaque characterization & ++ & + \\
\hline Assessment of SB ostium in the pullback of $\mathrm{MB}$ & $\mathrm{x}$ & ++ \\
\hline Determination of landing zone and stent length & ++ & ++ \\
\hline \multicolumn{3}{|l|}{ During stent implantation } \\
\hline Guidance of position of the guide wire towards the SB & $\mathrm{x}$ & ++ \\
\hline \multicolumn{3}{|l|}{ Post procedure } \\
\hline Evaluation of stent dimensions according to flow conservation law & ++ & ++ \\
\hline Stent under-expansion & ++ & ++ \\
\hline Edge dissection & + & ++ \\
\hline ISA & + & ++ \\
\hline \multicolumn{3}{|l|}{ Anatomic sub groups } \\
\hline LM ostial lesion & ++ & + \\
\hline
\end{tabular}

OCT and IVUS guidance results showed similar clinical outcomes, and seem to contribute to favourable clinical outcomes compared with CAG guidance only. IVI is more useful in subgroup of patients with complex lesions-(Long lesions, bifurcation and CTO) who have a higher risk of events. OCT should be considered for luminal assessment and stent related morphology in more complex cases in which CAG interpretation remaining uncertain. Since OCT and IVUS have distinct features, these techniques are complementary and should be considered carefully for each patient based on the merits and demerits of each technique.

\section{OCT New Algorithm-MLD MAX}

New Algorithm is proposed by light lab study investigators to implement simple 
work flow chart to make it simple and convenient to use while performing OCT guided interventional procedures.

Each OCT run serves a separate purpose. The pre-PCI run helps determine the PCI strategy, and the post-PCI run allows for optimization of the stent as needed

1) Pre-PCI OCT-Strategize (MLD)

a) Morphology

b) Length

c) Diameter

2) Post-PCI OCT-Optimize (MAX)

a) Medial dissection

b) Apposition

c) eXpansion

1) Pre-PCI OCT assessment-Strategize (MLD) (Figure 28)

1a) Morphology This new algorithm helps a beginner to interpret in a simple way. According to this classification pre-OCT run useful to assess requirement of plaque modification of the vessel before stenting. The morphology of a coronary artery (Figure 29) includes lipid plaque, fibrotic plaque, and calcium (mild, moderate and severe-Table 6) or sometimes any of the combination to decide direct stenting or plaque modification with complaint or non-complaint balloons (regular NC/OPN NC), Atherectomy (rotational/orbital) or IVL or ELCA followed by stenting (Table 7).

1b) Length: For selecting the landing zones-Visually scan for largest luminal area, Place landing zones in healthy tissue (i.e. EEL visualization). In the absence of EEL to represent healthy tissue find the largest lumen to avoid areas of TCFA or lipid pools so as to not land your stent edge in these high risk areas (Figure 30 ). Length of the stent is equal to the length between proximal and distal landing zones.

Table 6. Classification of calcium.

\begin{tabular}{ccc}
\hline & Calcium & \\
\hline Deep Calcific plaque & Calcific Nodule & Superficial Calcific plaque \\
\hline $\begin{array}{c}\text { Presence of thick fibrotic cap, } \\
\text { Non-Luminal Traditional }\end{array}$ & $\begin{array}{c}\text { Superficial calcium, Luminal } \\
\text { Protrusive, Attenuation present } \\
\text { Techniques-NC }\end{array}$ & $\begin{array}{c}\text { Minimal to no fibrotic layer, } \\
\text { depth of calcium likely }\end{array}$ \\
Balloons/Scoring/cutting & from thrombogenic surface. & measurable. \\
\hline
\end{tabular}

Table 7. OCT-based CVI score.

$\begin{array}{cl}\text { Angle } & \leq 180^{\circ} \longrightarrow 0 \text { Point } \\ & >180^{\circ} \longrightarrow 2 \text { Point } \\ \text { Thickness } & \leq 0.5 \mathrm{~mm} \longrightarrow 0 \text { Point } \\ & >0.5 \mathrm{~mm} \longrightarrow 1 \text { Point } \\ \text { Length } & \leq 5 \mathrm{~mm} \longrightarrow 0 \text { Point } \\ & >5 \mathrm{~mm} \longrightarrow 1 \text { Point }\end{array}$

Total Score 0 to 4 points 


\section{OCT Algorithm | MLD MAX}

Pre-PCI OCT | Strategize

\begin{tabular}{|c|c|}
\hline MORPHOLOGY & LENGTH \\
\hline If High Calcium & Select Landing Zones \\
\hline $\begin{array}{l}>180 \text { degrees, and } \\
>0.5 \mathrm{~mm} \text { thickness, and }\end{array}$ & $\begin{array}{l}\text { Visually scan for largest } \\
\text { luminal area }\end{array}$ \\
\hline $\begin{array}{l}>5 \mathrm{~mm} \text { in length } \\
\text { Common Practice: } \\
\mathrm{NC} \text { Balloon, IVL }\end{array}$ & $\begin{array}{l}\text { Place landing zones in } \\
\text { healthy tissue (i.e. EEL } \\
\text { visualization) }\end{array}$ \\
\hline $\begin{array}{l}\text { Cutting/Scoring Balloon, or } \\
\text { Atherectomy }\end{array}$ & $\begin{array}{l}\text { Note: In the absence of EEL to represent } \\
\text { healthy tissue find the largest lumen to } \\
\text { avoid areas of TCFA or lipid pools so as to } \\
\text { not land your stent edge in these high risk } \\
\text { areas }\end{array}$ \\
\hline & Adjust to stent length \\
\hline
\end{tabular}

DIAMETER

Measure Vessel Diameter

Take EEL measurements at each reference (lumen if EEL not visible)

Choose Stent Diameter

Use the distal reference measurement to select stent diameter

EEL: round down to nearest stent size. Lumen: round up

Choose Post Dilation Balloon Diameter

Distal Balloon: Use distal reference measurement Proximal Balloon: Use proximal reference measurement

Figure 28. Pre PCI OCT strategize.

\section{OCT Image Interpretation}

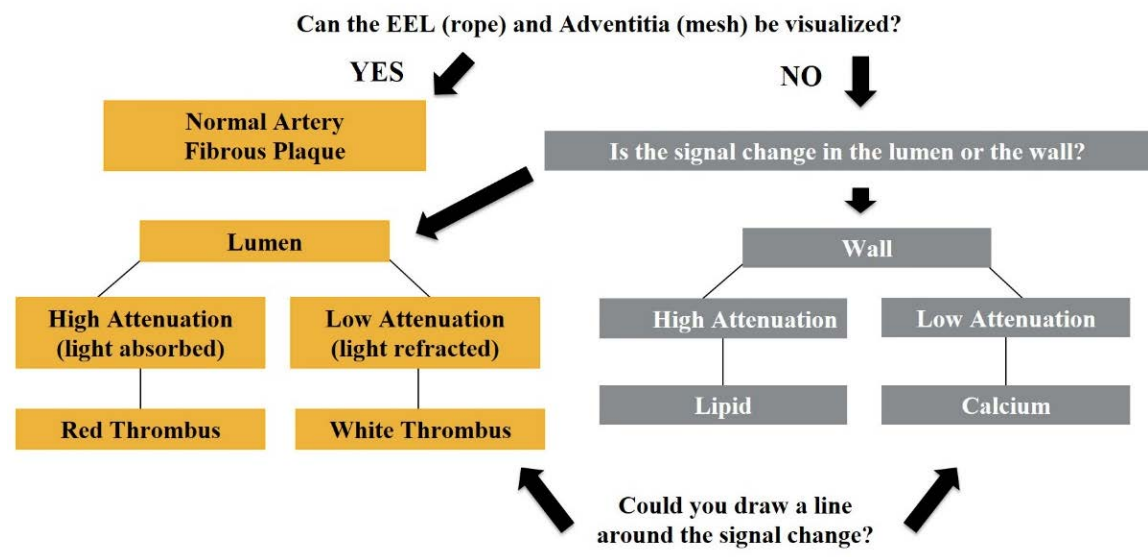

Figure 29. Morphological assessment by OCT.
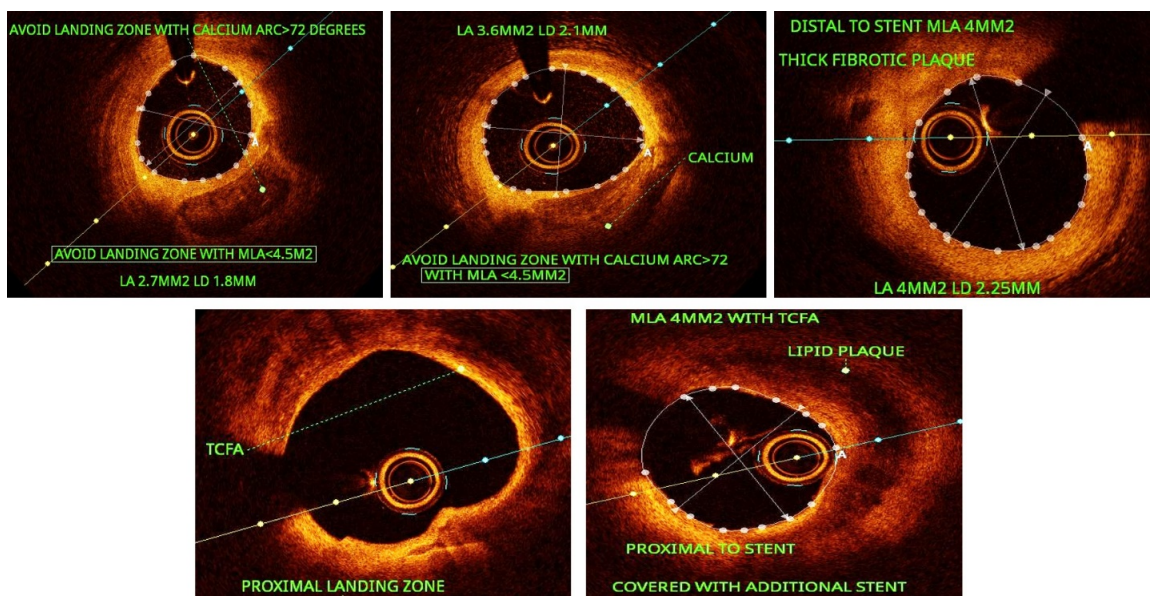

Figure 30. Assessment of lesion length, choose proper landing zones with minimal plaque burden or plaque free zone.

1c) Diameter: To measure vessel diameter, take EEL measurements at each reference (lumen if EEL not visible), and to choose stent diameter, use the distal reference measurement to select stent diameter (Figure 31). 

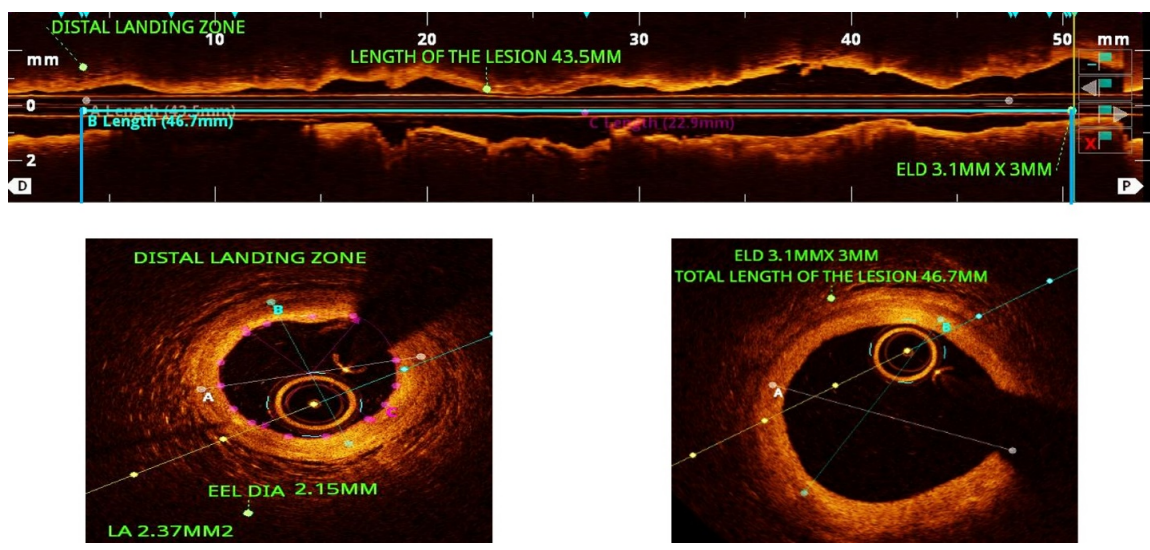

Figure 31. Assessment of diameter based upon EEL/luminal (figure with luminal areas and landing zones).

\section{OCT Algorithm | MLD MAX}

\section{Post-PCI OCT | Optimize}

MEDIAL DISSECTION

Address Significant

Dissection

Dissection penetrates

medial layer, and is greater

than 1 quadrant arc

Common Practice:

Place additional stent

(particularly for distal

dissections)

APPOSITION
Address Gross
Malapposition
Longer than $3 \mathrm{~mm}$, and
$\geq 0.3 \mathrm{~mm}$ from wall
Common Practice:
Dilate with semi-compliant
balloon at low pressure

\section{XPANSION}

Confirm Expansion

$\geq 80 \%$ acceptable $(\geq 90 \%$

optimal)

Common Practice:

If not achieved, post-dilate with non-compliant balloon

Figure 32. Post PCI OCT assessment-Optimize (MAX).

EEL: Round down to nearest stent size. Lumen: round up to choose post dilation balloon diameter, for distal balloon, use distal reference measurement and for proximal balloon use proximal reference measurement.

EEL Measurements:

1) Average two perpendicular EEL measurements

2) Round down to the next quarter size, unless already at a stent size

Lumen Measurements:

1) Use automatic measurements at distal reference

2) Round up to the next quarter size, even if already at a stent size

2) Post-PCI OCT-Optimize (MAX)

2a) Medial dissection: if dissection penetrates medial layer, and is greater than 1 quadrant arc that is to be corrected by placing additional stent (particularly in LM or ostial LAD/LCX or distal dissections).

2b) Apposition: If the gross mal apposition is $\geq 0.3 \mathrm{~mm}$ from wall and longer than $3 \mathrm{~mm}$, it should be corrected by dilating with semi complaint balloon at low pressure matching to vessel size.

2c) eXpansion: $\geq 80 \%$ expansion of the stent is acceptable and $\geq 90 \%$ expansion is optimal with reference to proximal and distal diameters. If not achieved, post dilate with non-complaint balloon, or high pressure OPN NC balloon (Figure 32). 


\section{Conclusion}

IVI is very useful in complex PCI in identifying plaque morphology, choosing plaque modification strategy, assessing adequacy of plaque modification, selecting proper devise size (diameter and length) in order to cover diseased segment, and choosing proper landing zones. Post PCI intravascular imaging identifies predictors of DES outcomes mainly under-expansion, edge dissection, mal apposition and helps in rectifying these issues. IVI guided PCI is associated with significantly reduced stent thrombosis and MACE rate compared to angio guided PCI.

\section{Conflicts of Interest}

The author declares no conflicts of interest regarding the publication of this paper.

\section{References}

[1] Ali, Z.A., Karimi Galougahi, K., Maehara, A., Shlofmitz, R.A., Ben-Yehuda, O., Mintz, G.S. and Stone, G.W. (2017) Intracoronary Optical Coherence Tomography 2018: Current Status and Future Directions. JACC: Cardiovascular Interventions, 10, 2473-2487.

[2] Imola, F., Mallus, M.T., Ramazzotti, V., Manzoli, A., Pappalardo, A., Di Giorgio, A., Albertucci, M. and Prati, F. (2010) Safety and Feasibility of Frequency Domain Optical Coherence Tomography to Guide Decision Making in Percutaneous Coronary Intervention. EuroIntervention, 6, 575-581. https://doi.org/10.4244/EIJV6I5A97

[3] Kubo, T., Akasaka, T., Shite, J., Suzuki, T., Uemura, S., Yu, B., Kozuma, K., Kitabata, H., Shinke, T., Habara, M. and Saito, Y. (2013) OCT Compared with IVUS in a Coronary Lesion Assessment: The OPUS-CLASS Study. JACC: Cardiovascular Imaging, 6, 1095-1104.

[4] Zhang, J., Gao, X., Kan, J., Ge, Z., Han, L., Lu, S., Tian, N., Lin, S., Lu, Q., Wu, X. and Li, Q. (2018) Intravascular Ultrasound versus Angiography-Guided Drug-Eluting Stent Implantation: The ULTIMATE Trial. Journal of the American College of Cardiology, 72, 3126-3137. https://doi.org/10.1016/j.jacc.2018.09.013

[5] Hong, S.J., Kim, B.K., Shin, D.H., Nam, C.M., Kim, J.S., Ko, Y.G., Choi, D., Kang, T.S., Kang, W.C., Her, A.Y. and Kim, Y.H. (2015) Effect of Intravascular Ultrasound-Guided vs Angiography-Guided Everolimus-Eluting Stent Implantation: The IVUS-XPL Randomized Clinical Trial. JAMA, 314, 2155-2163. https://doi.org/10.1001/jama.2015.15454

[6] Witzenbichler, B., Maehara, A., Weisz, G., Neumann, F.J., Rinaldi, M.J., Metzger, D.C., Henry, T.D., Cox, D.A., Duffy, P.L., Brodie, B.R. and Stuckey, T.D. (2014) Relationship between Intravascular Ultrasound Guidance and Clinical Outcomes after Drug-Eluting Stents: The Assessment of Dual Antiplatelet Therapy with Drug-Eluting Stents (ADAPT-DES) Study. Circulation, 129, 463-470. https://doi.org/10.1161/CIRCULATIONAHA.113.003942

[7] Garcìa-Garcìa, H.M., Gogas, B.D., Serruys, P.W. and Bruining, N. (2011) IVUS-Based Imaging Modalities for Tissue Characterization: Similarities and Differences. The International Journal of Cardiovascular Imaging, 27, 215-224. https://doi.org/10.1007/s10554-010-9789-7 
[8] Metz, J.A., Yock, P.G. and Fitzgerald, P.J. (1997) Intravascular Ultrasound: Basic Interpretation. Cardiology Clinics, 15, 1-15. https://doi.org/10.1016/S0733-8651(05)70314-3

[9] Pinto, F.J. and Moniz, A.P. (n.d.) Value of Preintervention Imaging before Interventions Balloon Coronary Angioplasty.

[10] Fitzgerald, P.J., St Goar, F.G., Connolly, A.J., Pinto, F.J., Billingham, M.E., Popp, R.L. and Yock, P.G. (1992) Intravascular Ultrasound Imaging of Coronary Arteries. Is Three Layers the Norm? Circulation, 86, 154-158.

[11] Prati, F., Romagnoli, E., Gatto, L., La Manna, A., Burzotta, F., Ozaki, Y., Marco, V., Boi, A., Fineschi, M., Fabbiocchi, F. and Taglieri, N. (2020) Relationship between Coronary Plaque Morphology of the Left Anterior Descending Artery and 12 Months Clinical Outcome: The CLIMA Study. European Heart Journal, 41, 383-391.

[12] Xing, L., Yamamoto, E., Sugiyama, T., Jia, H., Ma, L., Hu, S., Wang, C., Zhu, Y., Li, L., Xu, M. and Liu, H. (2017) EROSION Study (Effective Anti-Thrombotic Therapy without Stenting: Intravascular Optical Coherence Tomography-Based Management in Plaque Erosion): A 1-Year Follow-Up Report. Circulation: Cardiovascular Interventions, 10, e005860.

[13] Mintz, G.S. (2015) Intravascular Imaging of Coronary Calcification and Its Clinical Implications. JACC: Cardiovascular Imaging, 8, 461-471.

https://doi.org/10.1016/j.jcmg.2015.02.003

[14] Nissen, S.E. and Yock, P. (2001) Intravascular Ultrasound: Novel Pathophysiological Insights and Current Clinical Applications. Circulation, 103, 604-616. https://doi.org/10.1161/01.CIR.103.4.604

[15] Jensen, L.O., Thayssen, P., Mintz, G.S., Maeng, M., Junker, A., Galloe, A., Christiansen, E.H., Hoffmann, S.K., Pedersen, K.E., Hansen, H.S. and Hansen, K.N. (2007) Intravascular Ultrasound Assessment of Remodelling and Reference Segment Plaque Burden in Type-2 Diabetic Patients. European Heart Journal, 28, 1759-1764. https://doi.org/10.1093/eurheartj/ehm175

[16] Gatto, L., Golino, M., Marco, V., La Manna, A., Burzotta, F., Fineschi, M., Ruscica, G., Di Giorgio, A., Boi, A., Romagnoli, E. and Albertucci, M. (2018) Role of Optical Coherence Tomography in Identifying Sub-Optimal Stent Positioning and Predicting Major Adverse Cardiac Events in a Comparative Study with Angiography: A CLIO-OPCI II Sub-Study. Coronary Artery Disease, 29, 384-388. https://doi.org/10.1097/MCA.0000000000000633

[17] Räber, L., Mintz, G.S., Koskinas, K.C., Johnson, T.W., Holm, N.R., Onuma, Y., Radu, M.D., Joner, M., Yu, B., Jia, H. and Meneveau, N. (2018) Clinical Use of Intracoronary Imaging. Part 1: Guidance and Optimization of Coronary Interventions. An Expert Consensus Document of the European Association of Percutaneous Cardiovascular Interventions. European Heart Journal, 39, 3281-3300. https://doi.org/10.1093/eurheartj/ehy285

[18] Ahn, J.M., Lee, P.H. and Park, S.J. (2016) Practical Based Approach to Left Main Bifurcation Stenting. BMC Cardiovascular Disorders, 16, 49. https://doi.org/10.1186/s12872-016-0227-1

[19] Kang, S.J., Ahn, J.M., Song, H., Kim, W.J., Lee, J.Y., Park, D.W., Yun, S.C., Lee, S.W., Kim, Y.H., Lee, C.W. and Mintz, G.S. (2011) Comprehensive Intravascular Ultrasound Assessment of Stent Area and Its Impact on Restenosis and Adverse Cardiac Events in 403 Patients with Unprotected Left Main Disease. Circulation: Cardiovascular Interventions, 4, 562-569.

[20] Amabile, N., Rangé, G., Souteyrand, G., Godin, M., Boussaada, M.M., Meneveau, 
N., Cayla, G., Casassus, F., Lefèvre, T., Hakim, R. and Bagdadi, I. (2016) Optical Coherence Tomography to Guide Percutaneous Coronary. EIJ-D-20-01121.

[21] Legutko, J., Yamawaki, M., Costa, R.A. and Costa, M.A. (2015) IVUS in Bifurcation Stenting: What Have We Learned? EuroIntervention, 11, V55-V58.

[22] Hoye, A. (2017) The Proximal Optimisation Technique for Intervention of Coronary Bifurcations. Interventional Cardiology Review, 12, 110.

[23] Takagi, T., Stankovic, G., Finci, L., Toutouzas, K., Chieffo, A., Spanos, V., Liistro, F., Briguori, C., Corvaja, N., Albero, R. and Sivieri, G. (2002) Results and Long-Term Predictors of Adverse Clinical Events after Elective Percutaneous Interventions on Unprotected Left Main Coronary Artery. Circulation, 106, 698-702. https://doi.org/10.1161/01.CIR.0000024983.34728.5D

[24] Otsuka, F., Yasuda, S., Noguchi, T. and Ishibashi-Ueda, H. (2016) Pathology of Coronary Atherosclerosis and Thrombosis. Cardiovascular Diagnosis and Therapy, 6, 396. https://doi.org/10.21037/cdt.2016.06.01

[25] Gould, K.L., Lipscomb, K. and Hamilton, G.W. (1974) Physiologic Basis for Assessing Critical Coronary Stenosis: Instantaneous Flow Response and Regional Distribution during Coronary Hyperemia as Measures of Coronary Flow Reserve. The American Journal of Cardiology, 33, 87-94.

https://doi.org/10.1016/0002-9149(74)90743-7

[26] Oviedo, C., Maehara, A., Mintz, G.S., Araki, H., Choi, S.Y., Tsujita, K., Kubo, T., Doi, H., Templin, B., Lansky, A.J. and Dangas, G. (2010) Intravascular Ultrasound Classification of Plaque Distribution in Left Main Coronary Artery Bifurcations: Where Is the Plaque Really Located? Circulation: Cardiovascular Interventions, 3, 105-112.

[27] Watanabe, M., Uemura, S., Sugawara, Y., Ueda, T., Soeda, T., Takeda, Y., Kawata, H., Kawakami, R. and Saito, Y. (2014) Side Branch Complication after a Single-Stent Crossover Technique: Prediction with Frequency Domain Optical Coherence Tomography. Coronary Artery Disease, 25, 321-329. https://doi.org/10.1097/MCA.0000000000000091

[28] Balanescu, S. (2016) Fractional Flow Reserve Assessment of Coronary Artery Stenosis. European Cardiology Review, 11, 77. https://doi.org/10.15420/ecr/2016:24:2

[29] Mintz, G.S. (2016) IVUS in PCI Guidance. Journal of American College of Cardiology Expert Analysis.

https://www.acc.org/latest-in-cardiology/articles/2016/06/13/10/01/ivus-in-pci-guid ance

[30] Rusinova, R.P., Mintz, G.S., Choi, S.Y., Araki, H., Hakim, D., Sanidas, E., Yakushiji, T., Weisz, G., Mehran, R., Franklin-Bond, T. and Fahy, M. (2013) Intravascular Ultrasound Comparison of Left Main Coronary Artery Disease between White and Asian Patients. The American Journal of Cardiology, 111, 979-984.

https://doi.org/10.1016/j.amjcard.2012.12.014

[31] de la Torre Hernandez, J.M., Hernández Hernandez, F., Alfonso, F., Rumoroso, J.R., Lopez-Palop, R., Sadaba, M., Carrillo, P., Rondan, J., Lozano, I., Ruiz Nodar, J.M. and Baz, J.A. (2011) Prospective Application of Pre-Defined Intravascular Ultrasound Criteria for Assessment of Intermediate Left Main Coronary Artery Lesions: Results from the Multicenter LITRO Study. Journal of the American College of Cardiology, 58, 351-358. https://doi.org/10.1016/j.jacc.2011.02.064

[32] Jegere, S., Narbute, I. and Erglis, A. (2014) Use of Intravascular Imaging in Managing Coronary Artery Disease. World Journal of Cardiology, 6, 393.

https://doi.org/10.4330/wjc.v6.i6.393 
[33] Walsh, S.J., Cosgrove, C., Spratt, J.C. and Hanratty, C.G. (2019) A Technical Focus on Antegrade Dissection and Re-Entry for Coronary Chronic Total Occlusions: A Practice Update for 2019. Korean Circulation Journal, 49, 559. https://doi.org/10.4070/kcj.2019.0160

[34] Ahn, J.M., Kang, S.J., Yoon, S.H., Park, H.W., Kang, S.M., Lee, J.Y., Lee, S.W., Kim, Y.H., Lee, C.W., Park, S.W. and Mintz, G.S. (2014) Meta-Analysis of Outcomes after Intravascular Ultrasound-Guided versus Angiography-Guided Drug-Eluting Stent Implantation in 26,503 Patients Enrolled in Three Randomized Trials and 14 Observational Studies. The American Journal of Cardiology, 113, 1338-1347.

https://doi.org/10.1016/j.amjcard.2013.12.043

[35] Kim, B.K., Shin, D.H., Hong, M.K., Park, H.S., Rha, S.W., Mintz, G.S., Kim, J.S., Kim, J.S., Lee, S.J., Kim, H.Y. and Hong, B.K. (2015) Clinical Impact of Intravascular Ultrasound-Guided Chronic Total Occlusion Intervention with Zotarolimus-Eluting versus Biolimus-Eluting Stent Implantation: Randomized Study. Circulation: Cardiovascular Interventions, 8, e002592.

[36] Tan, Q., Wang, Q., Liu, D., Zhang, S., Zhang, Y. and Li, Y. (2015) Intravascular Ultrasound-Guided Unprotected Left Main Coronary Artery Stenting in the Elderly. Saudi Medical Journal, 36, 549. https://doi.org/10.15537/smj.2015.5.11251

[37] Zhang, J.-Q., et al. (2016) Application of Intravascular Ultrasound in Stent Implantation for Small Coronary Arteries. Journal of Clinical \& Invasive Cardiology, 3, 2-8.

[38] Park, S.J., Kim, Y.H., Park, D.W., Lee, S.W., Kim, W.J., Suh, J., Yun, S.C., Lee, C.W., Hong, M.K., Lee, J.H. and Park, S.W. (2009) Impact of Intravascular Ultrasound Guidance on Long-Term Mortality in Stenting for Unprotected Left Main Coronary Artery Stenosis. Circulation: Cardiovascular Interventions, 2, 167-177.

[39] Prati, F., Di Vito, L., Biondi-Zoccai, G., Occhipinti, M., La Manna, A., Tamburino, C., Burzotta, F., Trani, C., Porto, I., Ramazzotti, V. and Imola, F. (2012) Angiography Alone versus Angiography plus Optical Coherence Tomography to Guide Decision-Making during Percutaneous Coronary Intervention: The Centro per la Lotta contro l'Infarto-Optimisation of Percutaneous Coronary Intervention (CLI-OPCI) Study. EuroIntervention, 8, 823-829. https://doi.org/10.4244/EIJV8I7A125

[40] Kubo, T., Shinke, T., Okamura, T., Hibi, K., Nakazawa, G., Morino, Y., Shite, J., Fusazaki, T., Otake, H., Kozuma, K. and Ioji, T. (2017) Optical Frequency Domain Imaging vs. Intravascular Ultrasound in Percutaneous Coronary Intervention (Opinion Trial): One-Year Angiographic and Clinical Results. European Heart Journal, 38, 3139-3147. https://doi.org/10.1093/eurheartj/ehx351

[41] Ali, Z.A., Maehara, A., Généreux, P., Shlofmitz, R.A., Fabbiocchi, F., Nazif, T.M., Guagliumi, G., Meraj, P.M., Alfonso, F., Samady, H. and Akasaka, T. (2016) Optical Coherence Tomography Compared with Intravascular Ultrasound and with Angiography to Guide Coronary Stent Implantation (Ilumien III: Optimize PCI): A Randomised Controlled Trial. The Lancet, 388, 2618-2628. https://doi.org/10.1016/S0140-6736(16)31922-5 